\title{
ACCÉLÉRATION DU RÉGLAGE DE VITESSE DES TURBINES HYDRAULIQUES
}

\author{
par Jean DANIEL \\ Ingénieur des Arts et Métiers et de l'Ecole des Elèves Ingénieurs-Mécaniciens de la Marine.
}

(suite)

\section{CHAPITRE $V$}

\section{STABILITE DE VITESSE DANS LES INSTALLATIONS DE BASSE CHUTE}

\section{$1^{\circ}$ Etude qualifative. Equation}

Les installations de basse chute comportent des conduites d'alimentation de longueur relative. $2 \mathrm{~L}$

ment faible, de telle sorte que le temps $\frac{}{a}$ est lui-même réduit. Les inconnues $\varphi=\frac{\omega \mathrm{L}}{a}$ et $\gamma=\frac{\lambda L}{a}$ ne pourraient donc prendre des valeurs élevées que si ' $\omega$ et $\lambda$ étaient très grands, mais alors les difficultés de régulation que nous cherchons à étudier n'existeraient pas : $\lambda$ étant élevé, l'amortissement des oscillations serait considérable, quel que soit d'ailleurs $\omega$. Aussi est-il légitime d'admettre pour cette étude que les inconnues $\varphi$ et $\gamma$ restent faibles ef que leur combinaison $r=-y+i \varphi$ est dès lors assimilable à la fonction hyperbolique : th $r$ dont le développement en série s'écrit th $r=r-\frac{r^{3}}{3}+\frac{2 r^{5}}{15}-\frac{17 r^{7}}{315} \ldots$

Dans ces conditions, l'équation générale (17) s'écrit :

$$
\frac{r^{2}+(A+C) r+B}{\frac{r^{2}}{2}-\left(A-\frac{C}{2}\right) r-B}=-2 \rho r
$$

Au point de vue pratique, le temps $L / a$ étant faible, et à la limite nul, il est peu indiqué de le prendre pour référence. Nous adopterons dons. le temps $\theta=\frac{2 \rho L}{a}$, nettement plus élevé, et surtout beaucoup moins variable d'une installation à l'autre. Nous serons ainsi amenés à remplacer I'inconnue $r=\frac{p L}{a}$ par $r^{\prime}=p \theta=$ $2 \rho$ r. et à poser : $A^{\prime}=2 A \rho=\frac{K_{1} \theta}{\tau}$, $B^{\prime}=4 \rho^{2} B=\frac{K_{0} \theta^{2}}{\tau}, C^{\prime}=2 C \rho=\frac{\alpha^{\tau} \theta}{\tau}$.

\section{Limite de stabilité sans autoréglage}

Si nous négligeons $C^{\prime}$ (ou $C$ ), l'équation (19)

$$
\text { devient }: \frac{r^{2}+A r+B}{\frac{r^{2}}{2}-A r-B}=-2 \rho r
$$

et avec les notations indiquées au $\S 1$ ci-dessus :

$$
\frac{r^{\prime 2}+A^{\prime} r^{\prime}+B^{\prime}}{\frac{r^{\prime 2}}{2}-A^{\prime} r^{\prime}-B^{\prime}}=-r^{\prime}
$$

$c^{\prime}$ est-à-dire: $r^{\prime} 3+2\left(1-A^{\prime}\right) r^{\prime 2}+2\left(A^{\prime}-\right.$ $\left.B^{\prime}\right) r^{\prime}+2 B^{\prime}=0$

Cette équation coïncide avec celle donnée par M. ALMERAS sous le $n^{\circ} 12$ (Houille Blanche, p. 135) si l'on remplace $P^{\prime}$ par $y_{0} p \theta$ et $A^{\prime}$ et $B^{\prime}$ par les valeurs qu'ils représentent respectivement. L'assimilation de th $r$ à $r$ représente donc une simplification équivalente à I'hypothèse utilisée par M. ALMERAS, du mouvement en masse: toutes deux sont valides; elles restent acceptables tant que $r^{3 / 3}$ est négligeable devant $r$. 
Les conditions d'HURWITZ s'écrivent:

$$
0 \leqslant B^{\prime} \leqslant A^{\prime} \leqslant 1
$$$$
2\left(1-A^{\prime}\right) \quad\left(A^{\prime}-B^{\prime}\right) \geqslant B^{\prime}
$$

$c^{\prime}$ est-à-dire $: B^{\prime} \leqslant \frac{2 A^{\prime}\left(1-A^{\prime}\right)}{3-2 A^{\prime}}$

Si nous portons sur un diagramme $B^{\prime}$ en abscisses et $A^{\prime}$ en ordonnées, la limite indiquée par la condition (22) est représentée par un arc $\mathrm{d}^{\prime}$ hyperbole passant par l'origine et par le point

$$
\begin{aligned}
& \mathrm{A}^{\prime}=1 \\
& \mathrm{~B}^{\prime}=0 .
\end{aligned}
$$

Le maximum de $B^{\prime}$.correspond à :

$$
A^{\prime}=\frac{3-\sqrt{3}}{2} \div 0,634
$$

et a pour valeur: $B^{\prime}=2-\sqrt{3} \div 0,268$.

Cette limite est représentée sur la figure 20. Les combinaisons $A^{\prime}, B^{\prime}$ qui correspondent à des régimes stables sont figurées par des points compris entre l'arc d'hyperbole et l'axe des ordonnées; tous les points de cette zone et eux seuls satisfont à la fois aux conditions (21) ef (22).
On voit qu'il existe pour chacune des valeurs de $A^{\prime}$ (Efficacité de l'accéléromètre) un maximum que ne doit pas dépasser $B^{\prime}$ (Efficacité du tachymètre). Le paramètre $A^{\prime}$ luimême ne peut prendre que des valeurs comprises entre $O$ et 1 ; dans ces cas limites, $B^{\prime}$ devrait être nul, ce qui, on le sait, correspond à un délai de réglage infiniment grand (Chapitre $111, \S 3$ et figure 13). Remarquons encore que la zone de stabilité est telle que si le point $P$ représentant le régime de pleine puissance $s^{\prime} y$ trouve, l'arc de parabole OP de sommet $O$ et d'axe $O B$ qui représente les divers régimes réduits $\left(O<y_{0}<1\right)$. s'y trouve également. Les régimes réduits sont donc tous stables si le régime maximum l'est.

Ce sont là des propriétés générales que nous pourrions rétablir pour chacun des cas dont nous nous occuperons; nous ne le ferons pourtant pas, car elles sont aussi connues que faciles à établir

\section{$3^{\circ}$ Influence de l'autoréglage}

Si nous cessons de négliger l'autoréglage, l'équation (19) s'écrit :

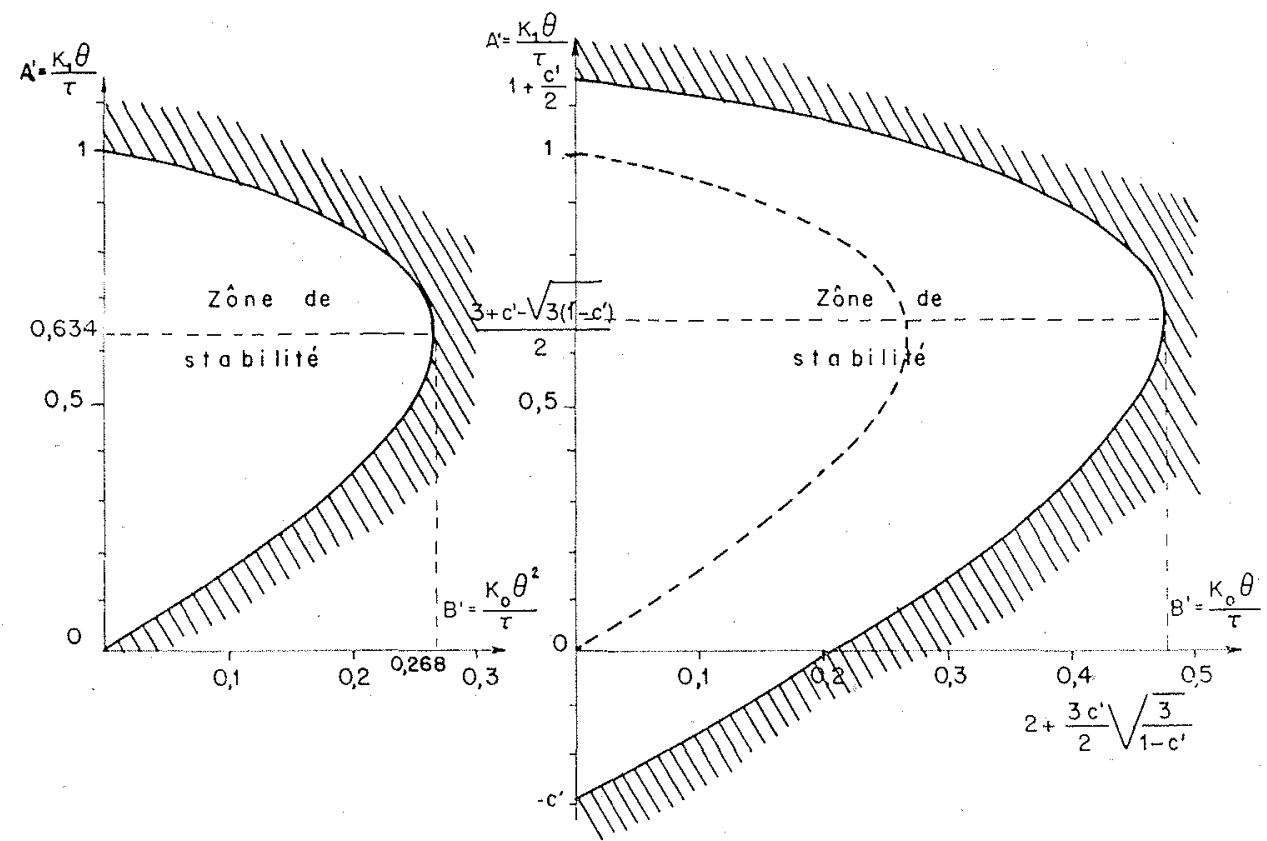

Fig. 20. - Limite de stabilité dans l'hypothèse du mou-

Fig. 21. - Limite de stabilité dans l'hypothèse du mouve. ment en masse avec autoréglage

vement en masse sans autoréglage.

$$
B^{\prime} \leqslant \frac{2 A^{\prime}\left(1-A^{\prime}\right)}{3-2 A^{\prime}}
$$

$$
\begin{aligned}
& B^{\prime} \leqslant \frac{\left(A^{\prime}+C^{\prime}\right)\left(2-2 A^{\prime}+C^{\prime}\right)}{3-2 A^{\prime}+C^{\prime}} \\
& C^{\prime}=\frac{\alpha \theta}{\tau}
\end{aligned}
$$

Ion a utilisé pour le tracé $C^{\prime}=0,3$ ) 


$$
\begin{aligned}
& \frac{r^{2}+(A+C) r+B}{\frac{r^{2}}{2} \cdot\left(A-\frac{C}{2}\right) r-B}=-2 \rho r \\
& \frac{r^{\prime 2}+\left(A^{\prime}+C^{\prime}\right) r^{\prime}+B^{\prime}}{r^{\prime 2}}=-r^{\prime} \\
& \frac{C^{\prime}}{2}-\left(A^{\prime}-\frac{C^{\prime}-B^{\prime}}{2}\right.
\end{aligned}
$$

et finalement $r^{\prime} 3+2\left(1-A^{\prime}+\frac{C^{\prime}}{2}\right) r^{\prime 2}+$

$2\left(\mathrm{~A}^{\prime}+\mathrm{C}^{\prime}-\mathrm{B}^{\prime}\right) \mathrm{r}^{\prime}+2 \mathrm{~B}^{\prime}=0$

Les conditions d'HURWITZ deviennent :

$$
0 \leq B^{\prime} \leq A^{\prime}+C^{\prime} \leq 1+\frac{3 C^{\prime}}{2}
$$

$$
2\left(1-A^{\prime}+\frac{C^{\prime}}{2}\right)\left(A^{\prime}+\vec{C}^{\prime}-B^{\prime}\right) \supseteq B^{\prime}
$$

c'est-à-dire :

$$
B^{\prime} \leq \frac{\left(A^{\prime}+C^{\prime}\right)\left(2-2 A^{\prime}+C^{\prime}\right)}{3-2 A^{\prime}+C^{\prime}}
$$

Pour chaque valeur de $C^{\prime}$, la limite imposée par la condition (25) est encore figurée par un arc d'hyperbole; celui-ci rencontre l'axe $O A^{\prime}$ aux points $A^{\prime}=-C^{\prime}$ et $A^{\prime}=1+\frac{C^{\prime}}{2}$ qui, lorsque $C^{\prime}$ est positit, sont extérieurs aux points homologues du cas précédent. Le maxi. mum de $B^{\prime}$ se présente lorsque

$A^{\prime}=\frac{3+C^{\prime}-\sqrt{3\left(1+C^{\prime}\right)}}{2}$ et a pour valeur :

$$
2+\frac{3 C^{\prime}}{2}-\sqrt{3\left(1+C^{\prime}\right)}
$$

toutes deux fonctions croissantes de $C^{\prime}$.

La zone de stabilité est donc plus érendue lorsque $C^{\prime}$ est positif. En particulier, on remarquera que l'accéléromètre $n^{\prime}$ est plus strictement nécessaire puisque, lorsque $A^{\prime}$ tombe à zéro, il reste possible de choisir des valeurs de $B^{\prime}$ telles que le régime soit stable. Nous avons représenté la zone de stabilité sur la figure 21 en adoptant pour le tracé $C^{\prime}=0,3$. Nous avons rappelé en mêmé temps la courbe de la figure $20\left(C^{\prime}=0\right)$.

\section{$4^{\circ}$ Etude quantitative}

Nous nous proposons avant tout, de réduire au minimum le délai de réglage, donc d'augmenter le plus possible les valeurs absolues des parties réelles des racines de la résolvante. Celleci est de la forme: $f\left(r^{\prime}\right)=0$. Or, pour qu'une équation $f\left(r^{\prime}\right)=0 \quad n^{\prime}$ ait aucune racine dont la partie réelle soit supérieure à une valeur négative donnée - $\gamma_{n}$, il faut et il suffit qu'une équation $F\left(r^{\prime \prime}\right)=0$, telle que $F\left(. r^{\prime \prime}\right)=$ $f\left(r^{\prime \prime}-\gamma_{\prime \prime}\right)$ satisfasse aux conditions d'HURVVITZ.

Si donc, à partir de l'équation (23), nous formons la fonction $F\left(r^{\prime \prime}\right)$ pour une valeur $\gamma_{0}$ donnée, nous pourrons en écrivant les conditions $d^{\prime}$ HURWITZ relatives à l'équation $F\left(r^{\prime \prime}\right)=0$, substituer à la «zone de stabilité » de la fig. 21 , la «zone de réglage rapide 》 de la figure 22 ; celle-ci est nécessairement tout entière intérieure à celle-là. En outre, si nous recommençons l'opération avec une valeur $\gamma_{1}$ plus élevée que $y_{0}$, nous obtiendrons une nouvelle zone intérieure à la précédente, et ainsi de suite. Pour une certaine valeur $\gamma_{. \prime}$ de $\gamma$, la zone se réduira à un point $M$ que nous appellerons optimum théorique, car aucune autre combinaison des paramètres $A^{\prime}$ et $B^{\prime}$ ne saurait procurer un



Fig. 22. - Détermination d'une zone de réglage rapide (amortissements supérieurs à une valeur donnée $y_{0}$ ).

N.-B. - Les lettres $P$ et $Q$ ont été oubliées dans la reproduction de la figure; elles désignent les deux points anguleux du contour bordé de hachures. 
amortissement de TOUS LES COMPOSANTS du mouvement libre égal ou supérieur à $\gamma_{M}$.

Un moyen plus rapide pour déterminer $M$ consiste à suivre les déplacements de $P$ et $Q$ ou de l'un seulement d'entre eux, lorsque $y$ varie; les courbes qu'ils décrivent aboutissent nécessairement en $M$ pour $\gamma=\gamma_{11}$. Nous allons donc rechercher ces points.

Si nous désignons par $f^{\prime}$ et $f^{\prime \prime}$ les dérivées première et seconde de $f$ par rapport à $r^{\prime}$, la fonction $F\left(r^{\prime \prime}\right)$ s'écrit (Développement de MacLaurin):

$F\left(r^{\prime \prime}\right)=r^{\prime \prime}+f^{\prime \prime}\left(--\gamma_{0}\right) r^{\prime \prime 2}+f^{\prime}\left(--\gamma_{0}\right) r^{\prime \prime}+f\left(-\gamma_{0}\right)$

Par suite, les conditions d'HURWITZ définissant la zone de réglage rapide de la figure 22 sont :

$$
\begin{array}{ll} 
& f^{\prime \prime}\left(-\gamma_{0}\right) \supseteq 0, f^{\prime}\left(-\gamma_{0}\right) \supseteq 0 \\
\text { et }: & f^{\prime \prime}\left(-\gamma_{0}\right) f^{\prime}\left(-\gamma_{0}\right) \supseteq f\left(-\gamma_{0}\right) \supseteq 0
\end{array}
$$

Dans la zone de réglage rapide, toutes sont satisfaites; à chacune de ses limites, l'une d'entre elles cesse de l'être. Celle-ci ne peut être l'une des conditions (26) prise isolément, car il faudrait alors pour que les deux conditions (27) continuent à être vérifiées, que les trois fonctions $f\left(-\gamma_{0}\right), f^{\prime}\left(-y_{0}\right)$ et $f^{\prime \prime}\left(-y_{0}\right)$ s'annulent en même temps. C'est dire que même dans ce cas, les limites du domaine restent définies par les limites des deux conditions (27). L'une
$f\left(-y_{0}\right)=O(28)$ correspond à la droite $P Q$ (La condition s'écrirait si nous l'explicitions:

$$
\left.B^{\prime} \supseteq A^{\prime} \gamma_{0}-\frac{\gamma_{0}{ }^{2}\left(1-\gamma_{0}\right)}{2\left(1+\gamma_{0}\right)}\right)
$$

et l'autre: $f^{\prime}\left(-\gamma_{0}\right) f^{\prime \prime}\left(--y_{0}\right)=f\left(-\gamma_{0}\right) \quad(29$ à la courbe. Quant aux points $P$ et $Q$, inter. sections de la droite et de la courbe, ils répondent à la fois aux deux équations (28) et (29) et par la suite à l'un des deux systèmes:

$\left\{\begin{array}{l}f\left(-y_{0}\right)=0 \\ f^{\prime}\left(-y_{0}\right)=0\end{array} \quad 30\left\{\begin{array}{l}f\left(-y_{0}\right)=0 \\ f^{\prime \prime}\left(-y_{0}\right)=0\end{array}\right.\right.$

L'élimination de $y_{0}$ dans chacun d'eux donne un lieu géométrique que parcourt $P$ ou $Q$; les deux lieux se rencontrent en $M$ optimum théorique.

\section{$5^{\circ}$ Optimum théorique}

L'optimum théorique est donc caractérisé par le fait que l'équation (23) a une racine triple - $\gamma^{\prime}$ et s'identifie donc à $\left(r^{\prime}+\gamma^{\prime}\right)^{3}=0$, $c^{\prime}$ est-à-dire

$$
\begin{aligned}
y^{\prime 3} & =2 B^{\prime} \\
3 y^{\prime \prime} & =2\left(A^{\prime}+C^{\prime}-B^{\prime}\right) \\
3 y^{\prime} & =2-2 A^{\prime}+C^{\prime}
\end{aligned}
$$

système qui, par élimination de $\gamma^{\prime}$, permet de

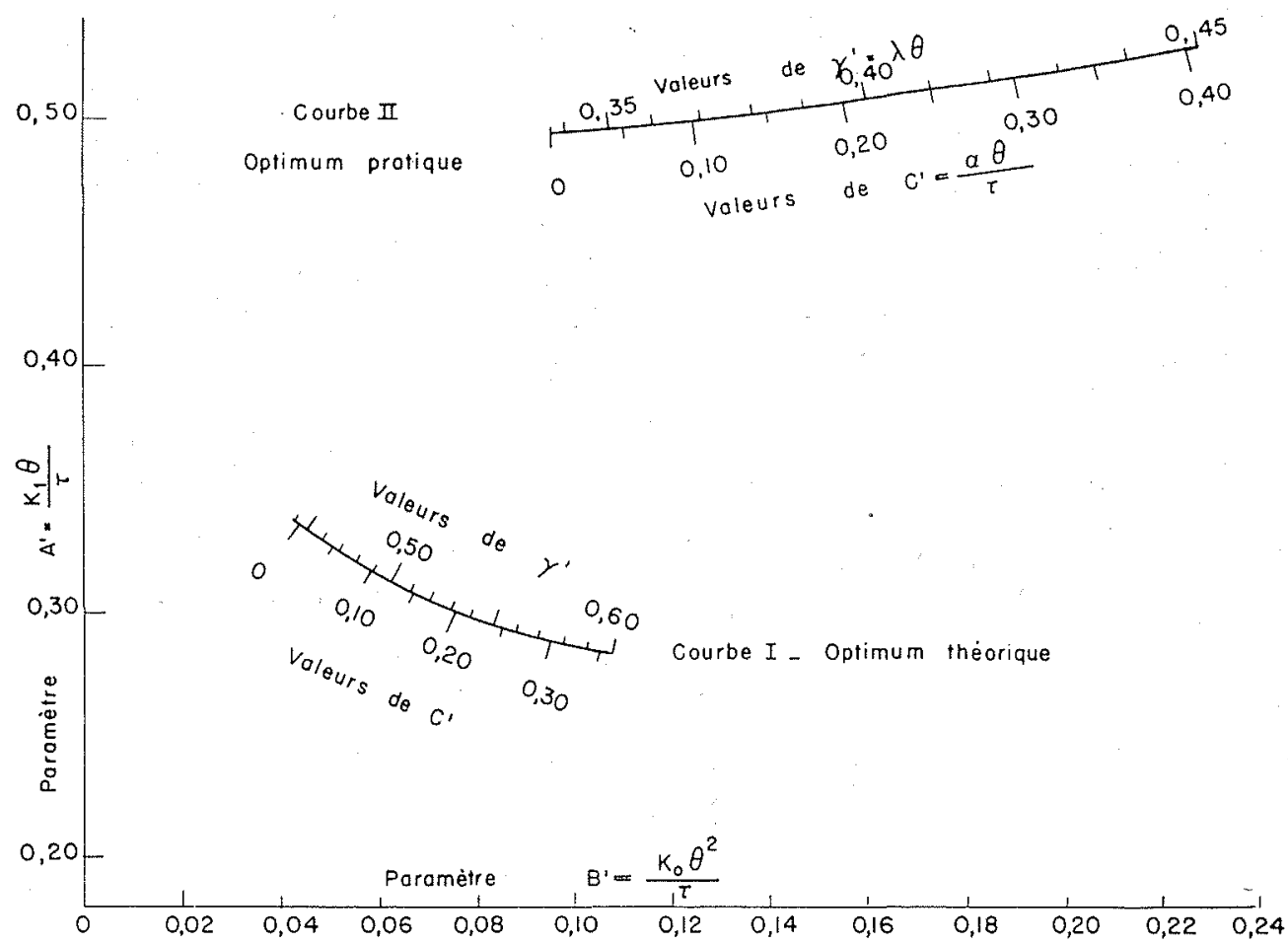

Fig. 23. - Réglage optimum dans l'hypothèse du coup de bélier en masse (pour l'optimum pratique la pulsation est $\omega=2 \lambda)$ 
déterminer $A^{\prime}$ et $B^{\prime}$ lorsque $C^{\prime}$ est donné. Toutefois, il est beaucoup plus simple en pratique d'utiliser le diagramme de la figure 23 (courbe 1) construit à partir des formes paramétriques :

$$
\begin{gathered}
A^{\prime}=\frac{\gamma^{\prime: 3}}{6}+\frac{\gamma^{\prime}}{2}-y^{\prime}+\frac{2}{3} ; B^{\prime}=\frac{\gamma^{\prime 3}}{2} ; \\
C^{\prime}=\frac{\gamma^{\prime 3}}{3}+y^{\prime 2}+\gamma^{\prime}-\frac{2}{3}
\end{gathered}
$$

II offre l'avantage $d^{\prime}$ indiquer immédiatement en fonction de $C^{\prime}$, non seulement $A^{\prime}$ et $B^{\prime}$, mais encore la valeur $y^{\prime}$ caractéristique de l'amortissement.

\section{$6^{\circ}$ Optimum relatif ef Optimum pratique}

Nous avons rappelé au Chapitre 11 et concrétisé sur la figure 12 le fait que, dans les mouvements pendulaires, le retour le plus rapide n'est pas réalisé pratiquement par l'amortissement critique, mais par un régime oscillant moins amorti, tel cependant que la première oscillation reste seule notable. Dans le réglage étudié ici, nous aurons intérêt à nous placer, dans les mêmes conditions, autant qu'il nous sera possible. Toutefois, l'équation étant du troisième ordre, et non plus du second, nous aurons toujours un mouvement apériodique superposé à l'oscillation, il semble indiqué de choisir les paramètres de telle sorte que les amortissements de ces deux composants soient égaux. Nous appellerons « optimum relatif » toute combinaison $A^{\prime}, B^{\prime}, C^{\prime}$ qui réalise cette égalité. Parmi les combinaisons ainsi retenues nous nommerons «optimum pratique » celle qui offrira une pul. sation assez faible pour que la première oscillation seule soit notable. II y a ici, nous I'avons déjà dit, une part de subjectivité : elle se manifeste par la fixation de l'écart résiduel que l'on accepte de négliger, fixation dont dépend le rapport $\frac{\varphi^{\prime}}{y^{\prime}}$ de la pulsation au facteur d'amortis sement. La relation entre ce rapport et l'écart négligeable est facile d̀ établir. Le rapport de l'écart maximum à celui de même sens qui le suit lavec un intervalle de temps égal à une pseudo-période) a pour logarithme naturel: $\frac{2 \pi \gamma^{\prime}}{\varphi^{\prime}}$; la valeur de $\frac{\varphi^{\prime}}{\gamma^{\prime}}$ à adopter lorsqu'on fixe à $\varphi^{\varphi^{\prime}}$ le seuil des oscillations appréciables (l'écart maximum étant pris pour unité) est donc :

$$
\frac{\varphi^{\prime}}{\gamma^{\prime}}=\frac{2 \pi}{\log \frac{1}{\varepsilon}} \div \frac{2,725}{\operatorname{colog} \varepsilon}
$$

en désignant par : colog. $\varepsilon$ le logarithme vul. gaire changé de signe de $\varepsilon$. Dans notre calcul, nous poserons $\frac{\varphi^{\prime}}{\gamma^{\prime}}=\mathrm{n}$.

Les relations entre $A^{\prime}, B^{\prime}, C^{\prime}, \gamma^{\prime}$, et $n$ comprennent le système (30):

$$
f\left(-y^{\prime}\right)=f^{\prime \prime}\left(-y^{\prime}\right)=0
$$

qui exprime la réalisation de l'optimum relatif et en outre l'équation $f\left(-y^{\prime}+\varphi^{\prime} i\right)=0$ précisant l'optimum qualifié de pratique. Développée par rapport aux puissances de $\varphi^{\prime} i$, celleci s'écrit :

$$
\begin{array}{r}
\left(\varphi^{\prime} i\right)^{3}+f^{\prime \prime}\left(-\gamma^{\prime}\right)\left(\varphi^{\prime} i\right)^{2}+f^{\prime}\left(-\gamma^{\prime}\right) \varphi^{\prime} i+ \\
f\left(-\gamma^{\prime}\right)=0
\end{array}
$$

et se décompose en :

$$
\left\{\begin{array}{l}
\varphi^{\prime} i\left[\varphi^{\prime 2}-f^{\prime}\left(-\gamma^{\prime}\right) \mid=0\right. \\
\varphi^{\prime 2} f^{\prime \prime}\left(-\gamma^{\prime}\right)-f\left(-\gamma^{\prime}\right)=0
\end{array}\right.
$$

De ces deux équations, la seconde est incluse dans le système (30) et la première, $\varphi^{\prime}$ n'étant pas nul, se réduit à $\varphi^{\prime 2}=f^{\prime}\left(-y^{\prime}\right)$

Appliquons maintenant ces résultats (Equations 30 et 31 ) à l'équation (23); il vient:

$$
\begin{aligned}
& \gamma^{\prime 3}-2\left(1-A^{\prime}+\frac{C^{\prime}}{2}\right) y^{\prime 2}+2\left(A^{\prime}+C^{\prime}-\right. \\
& \left.B^{\prime}\right) y^{\prime}-2 B^{\prime}=0 \\
& 3 y^{\prime}=2-2 A^{\prime}+C^{\prime} \\
& n^{2} y^{\prime 2}=3\left(y^{\prime 2}-2\left(2-2 A^{\prime}+C^{\prime}\right) y^{\prime}+\right. \\
& 2\left(A^{\prime}+C^{\prime}-\right.
\end{aligned}
$$

d'où l'on tire

$$
\begin{aligned}
2-2 A^{\prime}+C^{\prime} & =3 y^{\prime} \\
2\left(A^{\prime}+C^{\prime}-B^{\prime}\right) & =\left(n^{2}+3\right) y^{\prime 2} \\
2 B^{\prime} & =\left(n^{2}+1\right) \gamma^{\prime \prime}
\end{aligned}
$$

et par suite :

$$
\begin{aligned}
& A^{\prime}=\frac{n^{2}+1}{6} y^{\prime}+\frac{n^{2}+3}{6} y^{\prime 2}-y^{\prime}+\frac{2}{3} \\
& B^{\prime}=\frac{n^{2}+1}{2} y^{\prime 3} \\
& C^{\prime}=\frac{n^{2}+1}{3} \gamma^{\prime}+\frac{n^{2}+3}{3} y^{\prime}+\gamma^{\prime}-\frac{2}{3}
\end{aligned}
$$

A titre d'exemple, la courbe 11 de la figure 23 indique pour l'optimum pratique les valeurs de $A^{\prime}$ et $B^{\prime}$ et celles de $\gamma^{\prime}$ en fonction de $C^{\prime}$ lorsqu'on admet $n=2$. 


\section{$7^{\circ}$ Application numérique : Oprimum}

Pour compléter notre étude, nous donnerons deux séries d'applications numériques. La première, concrétisée par la figure 24, montrera la signification de l'optimum pratique, et sa rela-
Pour tracer des courbes de variation de vitesse susceptibles d'être comparées les unes aux autres, nous devons adopter un ensemble-type de conditions initiales et nous y tenir pour les diverses études. Nous supposerons qu'au début



Fig. 24. - Variation de la vitesse à la suite d'une petite modification brusque de la puissance demandée $(\theta=1,5 ; \quad \tau=7,5)$

tion avec l'optimum théorique. Nous y tracerons trois courbes de variation de la vitesse au cours de la période transitoire qui suit un changement brusque mais faible de la puissance consommée. Bien entendu, les trois courbes répondront à un même système de valeurs de $\theta$, $\tau$ et $\alpha$, mais différeront par les choix de $K_{i j}$ et $K_{1}$; nous constaterons les modifications avec ces paramètres des éléments de la stabilité : délai de réglage et labilité.

Nous adopterons une valeur moyenne de l'inertie du rotor se chiffrant par $\tau=7,5$; nous supposerons une inertie de l'eau relativement élevée, mais que l'on rencontre pourtant dès maintenant, et qui, dans les installations $\grave{c}$ venir, sera sans doute dépassée: $\theta=1,5$; enfin, bien que a soit mal déterminé, nous pensons rester plus près de la réalité en lui attribuant la valeur I plutôt qu'en le négligeant.

\section{a) Cherchons d'abord l'optimum pratique avec} $n=2$

II vient : $\quad C^{\prime}=\frac{\alpha \theta}{\tau}=0,2$

d'où, d'après la figure 23: $y^{\prime}=0.396$; $A^{\prime}=0,506 ; B^{\prime}=0,155$

On en déduit :

$$
\begin{gathered}
\lambda=\frac{\gamma^{\prime}}{\theta}=0,264 ; \quad K_{1}=\frac{A^{\prime} T}{\theta}=2,53 ; \\
K_{0}=\frac{B^{\prime} \tau}{\theta^{2}}=0,517 \\
\text { (1) }=2 \lambda=0,528 .
\end{gathered}
$$

de la période troublée, la vitesse et la chute sont normales mais, que, par suite d'une variation brusque de puissance consommée, l'ouverture du vannage diffère de celle qui convient à la nouvelle puissance. La valeur de cet écart d'ouverture, si conformément à nos hypothèses il reste faible, ne modifiera pas la durée des diverses variations qui seront la conséquence du déréglage, mais seulement leurs amplitudes: celles-ci lui seront proportionnelles. Nous pren. drons donc pour unité la valeur relative $\varepsilon_{0}$ de l'écart initial d'ouverture et nous calculerons le rapport $==\frac{\xi}{\varepsilon_{t 1}}$ ou éventuellement d'autres rapports tels que

$$
Z=\frac{\zeta}{\varepsilon_{0}}, E=\frac{\varepsilon}{\varepsilon_{0}}
$$

La variation de vitesse s'exprime par la loi : $\equiv=\left(a_{1}+a_{2} \sin \omega t+a_{3} \cos \omega t\right) e^{\lambda t}$ où $a_{1}, a_{2}, a_{1}$ sont des constantes d'intégration que l'on calcule par exemple en exprimant que $=\quad$ et ses deux premières dérivées ont à l'instant initial $(t=0)$ les valeurs respectives:

$$
0, \frac{1}{\tau}, \frac{1}{\tau^{2}}\left(2 K_{1}-\alpha\right)
$$

Ces valeurs sont déduites du système dont l'équation (23) est la résolvante, en y remplaçant $\xi, \zeta$ et $\varepsilon$ par leurs valeurs initiales respectives $O, O$ et $\varepsilon_{0}$ 
Ce système comprend les équations:

$$
\begin{aligned}
& \tau \frac{d \xi}{d t}+a \xi=\zeta+u \\
& \frac{d \varepsilon}{d t}=-K_{1} \xi-K_{1} \frac{d \xi}{d t} \\
& u=\varepsilon+\frac{\zeta}{2}
\end{aligned}
$$

et en outre l'équation (14): $\frac{\zeta}{v}=-2 \rho$ th $r$, simplifiée et mise sous la forme $\frac{\zeta}{u}=-p \theta$

(Hypothèse du mouvement en masse)

traduction de $\zeta=-\theta \frac{\mathrm{d} u}{\mathrm{~d} t}$

qui, combinée avec (12) donne:

$$
\zeta=-\theta\left(\frac{d \varepsilon}{d t}+\frac{1}{2} \frac{d \zeta}{d t}\right)
$$

Par ailleurs, de la loi (32), nous tirons pour l'instant initial :

$(=)_{0}=a_{1}+a_{: 3}$

$\left(\frac{d \equiv}{d t}\right)_{11}=a_{2}(1)-\left(a_{1}+a_{3}\right) \lambda$

$\left(\frac{d^{2}=}{d^{2}}\right)_{0}=\left(a_{1}+a_{3}\right) \lambda^{2}-2 a^{2} \lambda \omega-a_{3} \omega^{3}$

II vient par suite: $a_{1}+a_{3}=0$

$$
\begin{gathered}
a_{2}=\frac{1}{\tau \omega} \\
\frac{1}{\tau^{2}}\left(2 K_{1}-\alpha\right)+\frac{2 \lambda}{\tau}+a_{::} \omega^{2}=0
\end{gathered}
$$

d'où : $a_{1}=-a_{3}=\frac{2 K_{1}-\alpha+2 \tau \lambda}{\tau^{2}\left(\omega^{2}\right.}$

et dans le cas étudié : $a_{1}=0,512 ; a_{2}=0,252$ :

$a_{:}=-0,512$.

ce qui achève de déterminer la loi de variation (32) et permet de la représenter graphiquement comme nous l'avons fait sur la figure 24 courbe $a$.

\section{b) Si aw liew d'admettre $n=2$, nous avions pris $n=4$}

Nous aurions obtenu les résultats suivants:

$\begin{array}{lll}y^{\prime}=0,274 & A^{\prime}=0,689 & B^{\prime}=0,175 \\ \lambda=0,183 & \omega=0,731 & K_{1}=3,445 \\ K_{0}=0,583 & a_{i}=-a_{i}=0,288\end{array}$

$a_{2}=0,183$.

qui correspondent à la courbe b de la fig. 24.

\section{c) par contre en choisissant $n=0$}

Nous aurions réalisé I'optimum théorique caractérisé par les éléments suivants:

$\begin{array}{lll}\gamma^{\prime}=0,532 & A^{\prime}=0,300 & B^{\prime}=0,0754 \\ \lambda=0,355 & K_{1}=1,5 & K_{10}=0,251\end{array}$

La loi de variation de la vitesse prend alors la forme :

$==\left(b_{1}+b_{2} t+b_{3:} t_{2}\right) e^{-\lambda t}$

et à l'instant initial, la vitesse et ses dérivées successives s'écrivent:

$(\equiv)_{0}=b_{1}=0$

$\left(\frac{d \equiv}{d t}\right)_{0}=b_{2}-\lambda b_{1}=\frac{1}{\tau}$

$\left(\frac{d^{2} \equiv}{d t^{2}}\right)_{13}=\lambda^{2} b_{1}-2 \lambda b_{2}+2 b_{3}=\frac{2 K_{1}-\alpha}{\tau^{2}}$

On a donc $\equiv=\left(1+\frac{2 K_{1}-c \cdot-2 \tau \lambda}{2 \tau} t\right) \frac{t}{\tau} e^{-\lambda t}$

et dans le cas particulier:

$\equiv=\frac{t+0,488 t^{2}}{7,5} e^{-0,355 t}$

équation à laquelle correspond la courbe $c$ de la figure 24 .

L'ensemble de ces trois courbes montre que le régime apériodique $c$ est nettement moins intéressant que les deux autres quant au délai de réglage. Des deux autres régimes, l'un (a) a été choisi de telle manière que les exploitants les plus difficiles jugent la seconde oscillation négligeable; I'autre au contraire de telle sorte que tous disent que la seconde oscillation atteint ou dépasse la limite de ce que l'on peut raisonnablement négliger. Or, les temps qui s'écoulent avant le premier retour à la vitesse normale dans ces deux cas sont l'un de 10 secondes, l'autre de plus de 7 ; si l'on adopte leur moyenne pour mesure du délai de réglage, chacun d'eux diffère de cette moyenne de $16 \%$. En d'autres termes, les appréciations extrêmes du délai s'écartent d'environ $16 \%$ de l'estimation moyenne : ceci nous parait suffisant pour établir la valeur pratique de la notion de délai que nous avons intro- 
duite. Elle ne souffre pas d'une indétermination comparable à celle à laquelle on se heurte lorsqu'on veut caractériser une variation qui suit une loi exponentielle simple; on sait que pour celle-ci, les divers auteurs adoptent tantôt la constante de temps, tantôt un de ses multiples : temps de réduction au $1 / 4$, au $1 / 10$, au $1 / 516$. Nous continuerons donc à employer la notion de délai chaque fois que nous le pourrons; précisons dès maintenant que nous adopterons régulièrement la mesure la plus sévère, celle qui répond à $n=2$.

\section{$8^{\circ}$ Applications numériques: influences des inerties $\theta$ de l'eau et $\tau$ du rotor}

Cette autre série d'applications numériques est concrétisée par la figure 25 . Nous avons tracé là des courbes de variation de vitesse, comparables entre elles, en ce. sens qu'elles correspondent toutes à l'optimum pratique $(n=2)$; elles diffèrent par les valeurs des inerties $\theta$ et $\tau$ de l'eau et du rotor. tie du rotor, et atteint $\tau=10$ qui reste cependant dans les limites habituelles. Conservant alors l'inertie de l'eau du cas précédent $(\theta=3)$, nous avons tracé la courbe $b$ de la figure 25. La comparaison de ce tracé au tracé a de la même figure montre que l'accroissement de $33 \%$ de l'inertie du rotor est à peu près sans effet sur la durée du réglage, et certainement ne la diminue pas; par contre, il diminue la labilité de $18 \%$. Le seul intérêt de l'accroissement de l'inertie du rotor est donc la réduction des écarts de vitesse, exactement comme s'il s'agissait d'une machine sans régulateur.

Toutefois, comme il est admis très couramment que l'augmentation de l'inertie permet d'accélérer le réglage, nous donnerons une démonstration plus serrée du résultat qui vient d'apparaître. Rappelons d'abord comment les auteurs fondent l'opinion commune.

Ils établissent une condition de stabilité de la

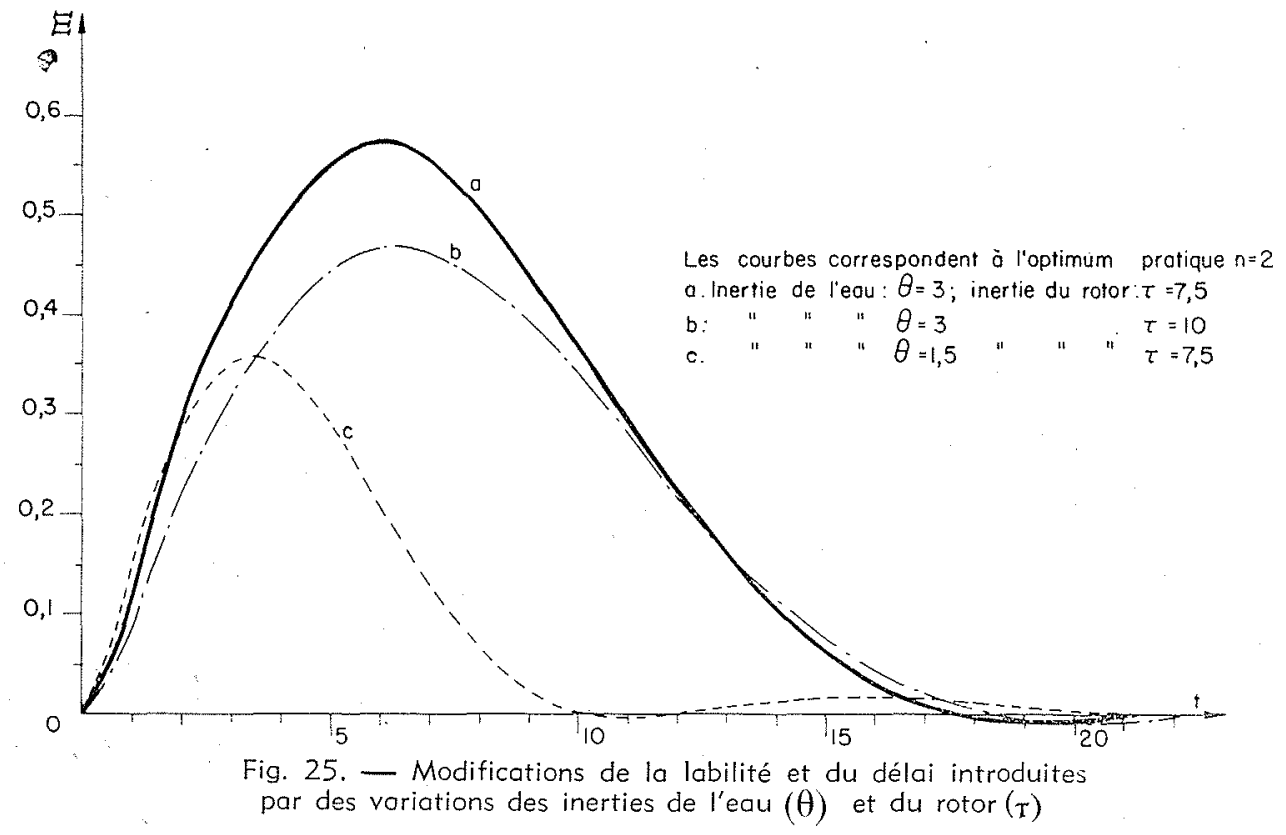

a) Nous avons doublé l'inertie de l'eau de telle sorte que le temps $\theta$ s'élève à 3 secondes, chiffre qui, dès maintenant, doit être envisagé pour certaines installations à l'étude. L'inertie du rotor a été maintenue : $\tau=7,5$. La courbe $a$ de la figure 25 indique la variation de vitesse dans ce cas. On voit en la comparant à la courbe a de la figure 24, reproduite à la figure 25 sous le repère $c$ que le fait de doubler $\theta$ a multiplié par 1,8 le délai de réglage et par 1,6 la labilité.

b) Nous avons ensuite élevé d'un tiers l'iner- forme $K_{0} \leqslant K \frac{\tau}{\theta 2}$ où $K$ est une constante dont ils précisent la valeur. De cette formule, ils concluent que toute augmentation de I dans une installation donnée ( $K$ et $\theta$ imposés) autorise une augmentation de $K_{0}$ dans le même rapport, et puisque ce paramètre représente dans la terminologie reçue la «PROMPTITUDE » du réglage, il semble évident que l'élévation de $\tau$ a pour conséquence dernière une régulation plus rapide. C'est cette évidence que nous voulons détruire. 
Au chapitre II, $\S 4$, nous avons qualifié de « semblables » des courbes qui pourraient se déduire l'une de l'autre par amplification ou réduction des échelles. Nous dirons de même deux régulations semblables si leurs réponses ì des causes perturbatrices semblables peuvent être représentées par un seul jeu de courbes: $\xi(t), \varepsilon(t), \zeta_{(t)}$ à condition seulement d'adopter pour la première (causes et effets) les échelles $\xi_{1}, \varepsilon_{1}, \zeta_{1}, \dagger_{1}$ et pour la seconde, d'autres échelles $\xi_{2}, \varepsilon_{2}, \zeta_{2}, t_{2}$. Cette définition n'est d'ailleurs que l'extension à notre problème de la similitude qu'emploie W.F. DURAND pour l'étude sur modèle des cheminées d'équilibre.

Afin de l'utiliser, il est intéressant de transformer les équations de la régulation:

$$
\begin{gathered}
\tau \frac{d \xi}{d t}+\alpha \xi=\frac{3}{2} \zeta+\varepsilon \quad(10 \text { bis } \\
\frac{d \varepsilon}{d t}=-K_{0} \xi-K_{1} \frac{d \xi}{d t} \\
\zeta=-\theta\left(\frac{d \varepsilon}{d t}+\frac{d}{2} \frac{d \zeta}{d t}\right)
\end{gathered}
$$

en posant : $t=\theta t^{\prime}$ et $\xi=\frac{\theta}{\tau} \xi^{\prime}$ elles prennent alors la forme réduite:

$$
\begin{aligned}
& \frac{\mathrm{d} \xi^{\prime}}{d t^{\prime}}+C^{\prime} \xi^{\prime}=\frac{3}{2} \zeta+\varepsilon \\
& \frac{d \varepsilon}{d t^{\prime}}=-B^{\prime} \xi^{\prime}-A^{\prime} \frac{d \xi^{\prime}}{d t^{\prime}} \\
& \zeta=-\frac{d \varepsilon}{d t^{\prime}}-\frac{1}{2} \frac{d x}{d t^{\prime}}
\end{aligned}
$$

où subsistent seulement les paramètres $A^{\prime}$ et $B^{\prime}$ par lesquels nous avons caractérisé les actions respectives de l'accéléromètre et du tachymètre et $C^{\prime}$ qui nous a servi à définir l'importance de l'autoréglage. Tous les systèmes qui admettent les mêmes paramètres $A^{\prime}$, $B^{\prime}$ et $C^{\prime}$ sont semblables; le passage de l'un à l'autre se fait en dilatant les temps comme les

$\theta$ et les écarts de vitesse comme les -, mais

sans changer les échelles de $\zeta$ et $\varepsilon$. Dès lors, sont semblables des causes perturbatrices telles que: des écarts instantanés mesurés par des valeurs égales de $\varepsilon$ (ouverture ou charge), $\xi^{\prime}$

(écarts de vitesse rapportés ou quotient - ), $\zeta$ (surpression) ; une variation de charge progressive appliquée tantôt à l'un, tantôt à l'autre de ces systèmes satisfait à la loi de similitude s'ils ont tous même temps caractéristique $\theta$. De telles causes ont sur les divers systèmes semblables des influences semblables. En particulier, les durées des troubles qu'elles apportent aux régulations semblables affectées de la même inertie $\theta$ de l'eau sont égales.

Par ailleurs, les effets sur un même système de deux causes dont l'une est déduite de l'autre par modification de toutes les amplitudes $(\xi, \zeta, \varepsilon)$ dans un même rapport a ont eux aussi des durées égales, car les équations réduites ne sont pas changées quand on y multiplie toutes les variations autres que $t^{\prime}$ par $a$.

Des régulations semblables adaptées à des installations où $\theta$ a la même valeur, répondraient donc dans le même temps, non seulement à des variations brusques de charge comme l'a fait apparaitre notre exemple numérique, mais encore à toutes autres perturbations qui seraient comparables. Tel serait entre autres, le cas de variations de charge imposées dans des temps égaux, soit linéairement, soit même suivant des lois représentées dans le temps par des courbes superposables par modification d'ordonnées dans un rapport constant. Ce serait aussi le cas de variations comparables d'autres paramètres tels que la position du réglage secondaire chargevitesse ou le niveau de la retenue, etc.

Précisément, lorsqu'on modifie une régulation par accroissements proportionnels de $\tau$ et $K_{0}$, $\theta$ restant constant, le nouvel ensemble réalisé soutient avec le premier la relation de similitude qui vient d'être étudiée, et qui est caractérisée par l'invariance du temps de réponse à chaque perturbation. On ne peut donc dire en aucune manière que la transformation en cause rend le réglage plus rapide, et c'est seulement par un abus de langage qu'on lui attribue une augmentation de «promptitude».

L'illusion est due au fait qu'à un écart de vitesse donné, correspond dans le nouvel ensemble un écart de charge plus grand; comme l'opéra. tion de réglage dure le même temps dans les deux cas, la variation de charge est plus rapide dans le second, bien que la variation de vitesse ne le soit pas. On doit donc dire, si l'on veut être clair : «A égalité d'écart initial de vitesse, les variations de vitesse sont également rapides $D$ ou encore: «A égalité d'écart de charge, les variations de charge sont également rapides », mais non: "A écarts initiaux de vitesse égaux, les vitesses de variation de charge sont différentes $\gg$.

Aussi l'inertie du rotor n'est-elle pas à choisir en considération de la rapidité d'action du régu- 
lateur, mais plutôt par exemple, en vue de limiter les à-coups dans les lignes électriques. Au cours d'une manœuvre de réglage, celles-ci doivent en effet transporter une puissance « synchronisante 》 successivement dans deux sens:

1) avant l'action des régulateurs, la variation de puissance consommée entraîne des variations des puissances livrées par les diverses usines proportionnelles aux inerties qu'elles mettent en jeu; il y a donc intérêt, pour limiter la variation de puissance transportée, à ne pas exagérer les différences d'inertie entre groupes.

2) lorsque les régulateurs les plus rapides ont seuls repris leur équilibre, ils délivrent, par le jeu des statismes, une puissance qui correspond à un surréglage et aide à l'accélération des groupes hydrauliques par un transfert de puissance «synchronisante ». Le surréglage sera d'autant moindre que l'écart de vitesse résiduel sera moindre, donc que l'inertie des groupes hydrauliques sera moindre. *

La considération des deux transferts de puissance successifs et de sens contraires peut donc être à l'origine d'un compromis aboutissant à la fixation de l'inertie des groupes hydrauliques.

Notons cependant que d'autres éléments au moins aussi importants interviennent dans la même détermination. En particulier, l'obligation de construire économiquement un groupe de puissance donnée à vitesse donnée empêche de modifier beaucoup l'inertie. Par ailleurs, la nécessité de limiter la vitesse d'emballement impose un minimum à l'inertie, du moins si l'on ne peut ou ne veut pas compter sur un déchargeur pour assurer la sécurité de la conduite. Aussi notre remarque concernant l'incidence de l'inertie du rotor sur la régulation n'a-t-elle d'autre but en définitive que de ramener à sa valeur vraie un argument risquant de pousser à la trop augmenter.

\section{CHAPITRE VI}

\section{STABILITE DE VITESSE DANS LES INSTALLATIONS DE HAUTE CHUTE}

\section{Méthode de l'érude qualitarive}

Nous ne pouvons plus adopter ici la simplifi. cation du chapitre précédent, où du fait de la longueur relativement faible de la conduite, nous pouvions assimiler la fonction th $r$ à la variable r. Deux avantages nous en revenaient: d'une part, nous pouvions substituer ò la résolvante transcendante une équation du troisième degré; d'autre part, les deux para. mètres caractérisant la conduite $o$ et $\mathrm{L} / \mathrm{a}$ n'intervenaient plus que par leur produit $\frac{\rho L}{a}=\frac{\theta}{2}$, ce qui simplifiait notablement la discussion. Maintenant, nous devrons nous tenir à l'équation transcendante à quatre paramètres: $A, B$,o et $L / a$, ou même cinq, si nous tenons compte de l'autoréglage $\alpha$; en fait, nous écarterons l'autoréglage qui compliquerait inutilement l'exposé, son influence ayant été suffisamment mise en valeur aux chapitres précédents, et n'ayant aucune raison de se manifester différemment en hautes chutes. Nous utiliserons donc l'équation (18) établie au chapitre IV:

$$
\frac{r^{2}+A r+B}{\frac{r^{2}}{2}-A r-B}=-2 \rho \text { th } r
$$

et nous la traiterons par une méthode analogue à celle de NYQUIST, les conditions d'HURWITZ ne pouvant être utilisées dans ce cas.

$$
\text { - } \rho \text { th } r \text {, par } Y \text { le quotient: } \frac{\begin{array}{l}
r^{2}+A r+B \\
\frac{r^{2}}{2}-A r-B
\end{array}}{\text { la fonction }}
$$

et par $R$ le rapport $X / Y$. Celui-ci sera l'analogue de la réponse de NYQUIST: $2 X$ représentera en effet la « réponse » de la conduite: $H_{(0)} / V_{(0)}$, aux variations de débit de pulsation (1) lorsque $r=\frac{i \omega L}{a}=i \varphi$; sans autre considération, l'équation (18) montre que la « réponse » correspondante de l'ensemble turbine-régulateur est : 1/2Y. D'après la justification et l'énoncé que nous avons donné de la méthode, il serait d'ailleurs assez indifférent d'utiliser $R$ ou son inverse $1 / R$; nous nous tiendrons cependant à la convention reçue, puisque rien ne justifierait une attitude contraire.

Nous tracerons donc la courbe de réponse :

$R_{0}=\frac{X_{0}}{Y_{11}}=-20 i+g \varphi \frac{\frac{\varphi^{2}}{2}+B+A \varphi i}{\varphi^{2}-B-A_{\varphi} i}(34)$

l'indice $O$ étant ajouté simplement pour signi. fier que, dans la variable: $r=-\gamma+\mathrm{i} \varphi$, la partie réelle - y a été prise nulle. Sa forme générale lorsque $A$ et $B$ sont positifs, est indiquée par la figure 26 qui a été établie en supposant $\rho=0,3, \quad A=0,44, \quad B=0,06$. On remarque les propriétés suivantes, que nous allons établir: lorsque $\varphi$ augmente à partir de zéro, et croît indéfiniment : 
1) la direction du rayon vecteur tourne d'un mouvement rétrograde continu dont l'amplitude est de $180^{\circ}$.

2) le module du rayon vecteur, nul avec $\varphi$, croît indéfiniment lorsque $\varphi$ tend vers $\frac{\pi}{2}$; le point figuratif atteint alors la droite de l'infini du côté des abscisses positives sur une première asymptote et passe brusquement au point symé. trique par rapport à l'origine ; il se rapproche ensuite de l'origine, le rayon vecteur continuant à tourner dans le même sens, puis passant à l'origine lorsque $\varphi$ atteint $\pi$, revient du côté des abscisses positives, et repart à l'infini lorsque $\varphi$ atteint $\frac{3 \pi}{2}$, dans les mêmes conditions que la première fois lorsque $\varphi$ atteignait $\frac{\pi}{2}$

3) Une nouvelle branche de courbe correspond à la variation de $\frac{3 \pi}{2}$ à $\frac{5 \pi}{2}$, etc. Le nombre des branches est infini ; elles tendent asymptotiquement à se confondre avec l'axe imaginaire.

4) la courbe ne rencontre donc l'axe des abscisses, en dehors de l'origine, qu'une seule fois.

Ces propriétés sont les conséquences immédiates des suivantes que seules nous démontrerons directement: l'argument de $Y_{0}$ croît $d^{\prime}$ une manière continue de $-\pi$ à $O$; et son module reste fini sans s'annuler. Le double de son affixe s'écrit :

$$
2 Y_{0}=-\frac{\varphi^{2}-B-A \varphi i}{\frac{\varphi^{2}}{2}+B+A \varphi i}
$$

Le numérateur est représenté (figure 27) par la parabole 1 dont le sommet est le point d'abscisse - B de l'axe réel, et le paramètre $A=12$. Le dénominateur est représenté par la parabole 11 dont le sommet est symétrique du précédent par rapport à l'origine, et dont le paramètre est $A$ ?. Les points $N$ et $D$ correspondant à une même valeur de $\varphi$ ont, au signe près, même ordonnée. Aussi, un moyen commode pour déterminer le point $D$ répondant à un point $N$ donné, consiste-t-il à mener une parallèle à l'axe des abscisses par $M$ symétrique de $N$ par rapport à $O$. Le point $M$ décrit la parabole III. Nous allons montrer que, lorsque $\varphi$ partant de $O$, croît de façon conti. nue et indéfinie, l'angle $\overline{\mathrm{DOM}}$, partant de $\mathrm{O}$ croît de façon continue de $O$ à $\pi$. Les valeurs extrêmes apparaissent immédiatement.

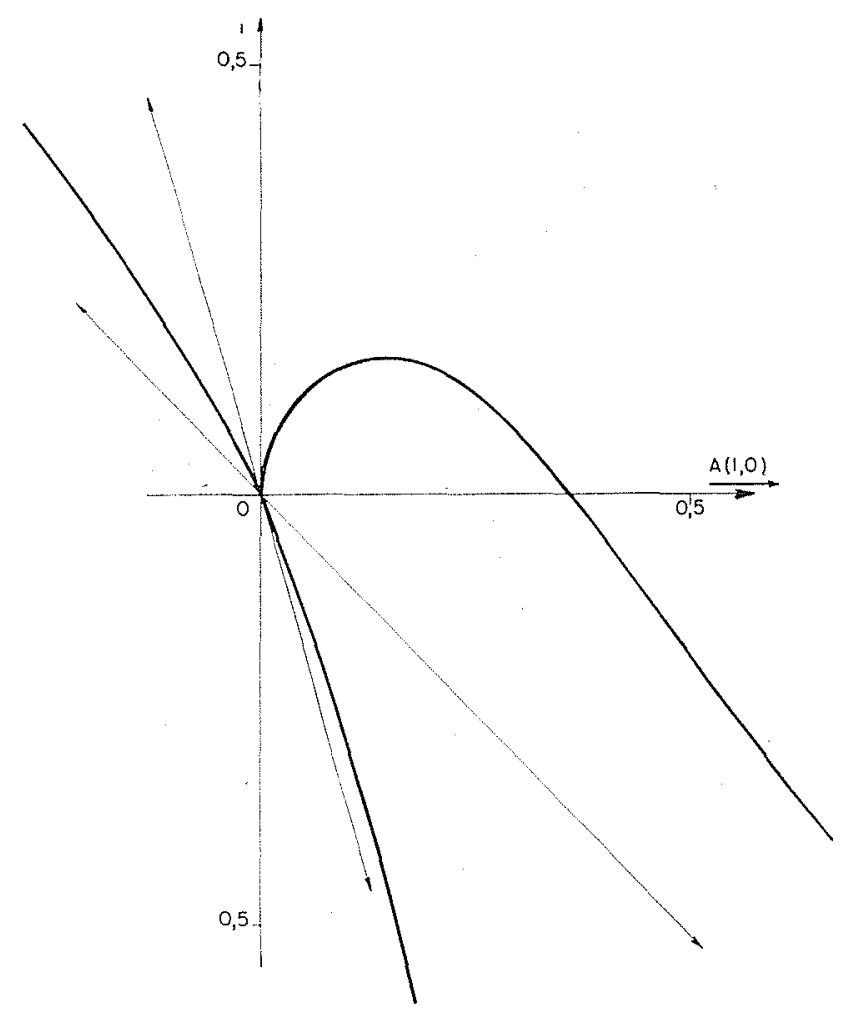

Fig. 26. - Exemple de courbe de réponse. On a adopté : $\rho=0,3 ; A=0,44 ; B=0,06$

II nous suffira donc de montrer qu'au passage $d^{\prime}$ une position quelconque $D M$ à une position infiniment voisine $D^{\prime} M^{\prime}$, la variation $\overline{M O M^{\prime}}$ - $\overline{\mathrm{DO} \mathrm{D}^{\prime}}$ est de même signe que $\triangle \varphi$. Or ceci est évident si l'on remarque

1) au delà de la position $M_{1} D_{1}$ pour laquelle $O D_{I}$ est tangente à la courbe 11 les deux angles à soustraire sont de signes contraires: leur différence est donc nécessairement de même signe que le premier, donc que $\triangle \varphi$.

2) En deçà :

a) la longueur $M M^{\prime}$ est supérieure à $D$ D' (Même écart d'ordonnées et pentes dans le rapport 2).

b) Le rayon vecteur $O M$ est plus court que OD. Ordonnées égales et abs. cisse de $M$ inférieure en valeur absolue à celle de D.).

c) L'angle du rayon vecteur avec la normale est moindre en $M$ (ou $M_{2}$ ) qu'en $D$ (ou $D_{2}$ ). Démontrons - le pour le cas de figure de $M$ qui exige le raisonnement le plus complet:

$$
\begin{aligned}
& 4<\sqrt{3} \text { car } \mathrm{OM}_{2}<O \mathrm{D}_{2} \text { idans } \\
& \text { les triangles rectangles } \\
& O \mathrm{M}_{2} \mathrm{~m}_{2} \text { et } \mathrm{O} \mathrm{D}_{2} \mathrm{~d}_{2} \text { ) }
\end{aligned}
$$




\section{$2<1$ car la sous-normale d'une parabole $m_{2} m^{\prime}$ : par exem ple, étant égale au demi- paramètre, $d_{2} d_{2}^{\prime}=2 m_{2} m^{\prime}$}

donc $2+4<1+3$

L'ensemble des trois propriétés $a, b c$ établit que, même lorsque $M D$ est au-dessous de $M_{1} D_{1}$. la différence $\overline{M O M^{\prime}}-\overline{\mathrm{DOD}}$ a même signe que $\triangle \varphi$. La mesure de l'angle $\overline{\mathrm{DOM}}$ croît donc continuellement de $O$ à $\pi$ lorsque $\varphi$ croît de manière continue et indéfinie à partir de zéro. Par suite, l'angle $\overline{N O D}$ décroit continuellement de $\pi$ à $O$ dans le même temps, et l'argument de $Y_{0}$ croît lui aussi constamment de $-\pi$ à $O$.

Quant au module de $Y_{0}$, il reste fini sans s'annuler, puisque ni: $O N$ ni $O D$ ne s'annule, et que, lorsque l'un d'eux croit indéfiniment, l'autre le fait aussi, leur rapport tendant alors vers 2 .

Ceci achève la démonstration des propriétés de la courbe de réponse relevées à l'examen de la figure 26 , tracée pour les valeurs positives des paramètres $A$ et - $B$.

Si l'on remplace $A$ par - A, le module de $Y_{0} n^{\prime}$ est pas modifié, mais son argument change de signe; la courbe de réponse présente donc dans ce cas, une disposition symétrique de celle de la figure 26 par rapport à I'axe imaginaire. $\mathrm{Si}$ I'on remplace $\mathrm{B}$ par - B, la modification de la courbe de réponse est plus compliquée; un raisonnement analogue à celui que nous avons fait à l'aide de la figure 27 montrerait qu'un changement de signe de $B$ est, au point de vue des propriétés générales, que nous utilisons, équivalent à un changement de signe de A.

\section{$2^{\circ}$ Condition de stabilité}

Nous étudierons d'abord le cas où $A$ et $B$ sont positifs. La fonction $Y$ n'a alors aucun zéro répondant à des valeurs de $r$ situées dans le demi-plan positif ou à ses frontières. La fonc. tion $X$ a un nombre infini de pôles, tous d'ordre I et correspondant aux valeurs purement imaginaires de la variable $r$. D'après ce que nous avons dit au chapitre 111 \$ 7 ci-dessus, le critère de NYQUIST n'est donc pas utilisable directement. Toutefois, comme il n'y a de pôles qu'aux frontières du contour $M M^{\prime} N N^{\prime}$ (fig. 17) et non à son intérieur, nous pourrons recourir à l'artifice qui consiste à utiliser un? contour $M_{1} M_{1}^{\prime} N N^{\prime}$ infiniment proche de $M M^{\prime}$ $N N^{\prime}$, et à la limite se confondant avec lui. La courbe de réponse $R_{0}=f(i \varphi)$ est alors remplacée par une courbe $R_{\varepsilon}=f(\varepsilon+i \varphi)$ où $\varepsilon$ est un nombre positif tendant vers zéro. La modification de forme provient du remplacement dans l'expression de $R$ du facteur th ( $i \varphi$ ) qui présente des discontinuités pour $\varphi=k \pi+\frac{\pi}{2}$ par le facteur th $(\varepsilon+i \varphi)$ qui ne diffère du précédent que par la substitution aux discontinuités de demi-cercles de rayon

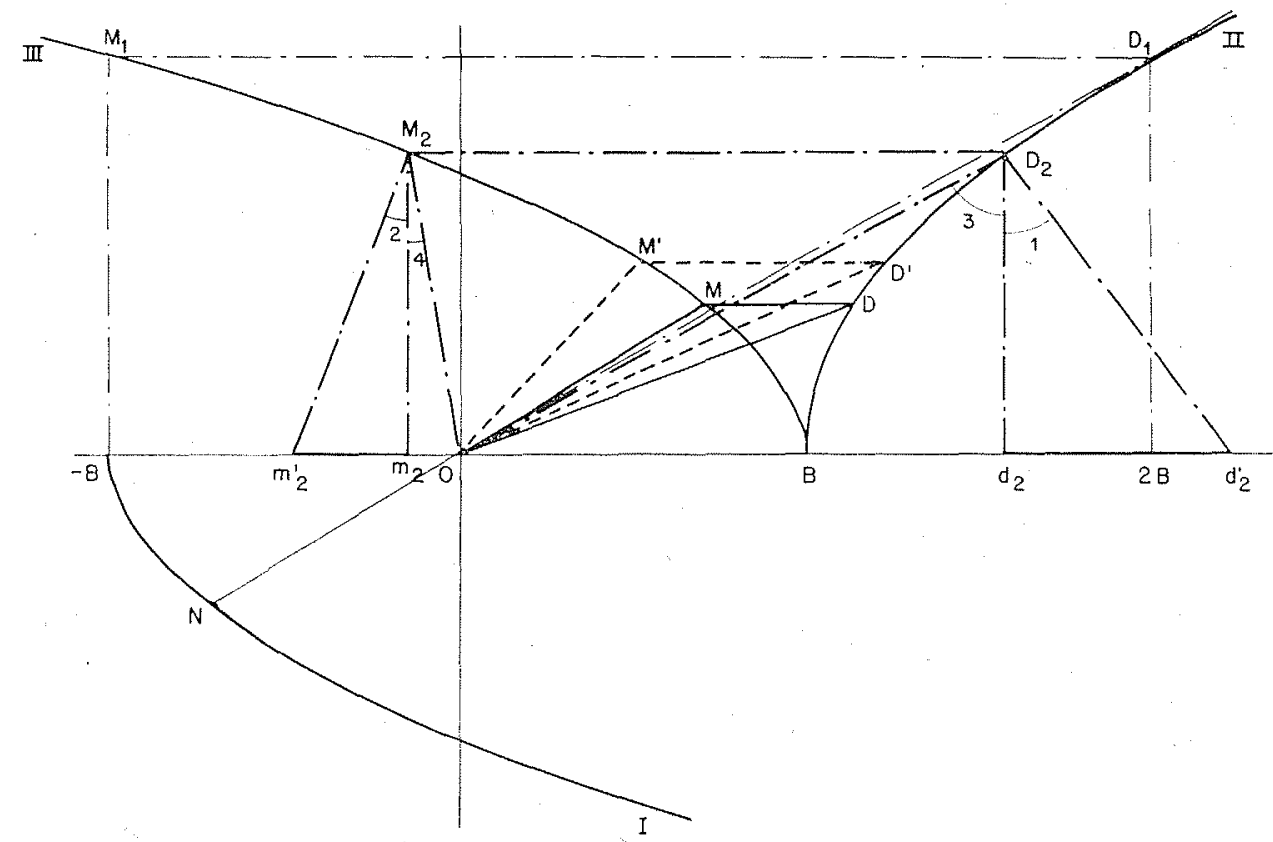

Fig. 27. - Démonstration de la biunivocité de la relation entre $\varphi$ et l'argument de $Y_{0}$. 
infini décrits dans le sens rétrograde; aussi la courbe $\mathrm{R}$ \& n'est-elle autre que la courbe de la figure 26, complétée par des demi-circonférences de rayon infini et de sens rétrograde comblant les discontinuités.

Cette courbe $R_{\varepsilon}$, doublée par symétrie autour de l'axe réel pour les valeurs négatives de $\varphi$, correspond à la portion $M_{1}^{\prime} M_{1}$ du contour $M_{1}^{\prime} M_{1} N N^{\prime} M_{1}^{\prime}$ (fig. 17) du demi-plan positif. A la portion $N N^{\prime}$ tout entière correspond le seul point $B$ d'abscisse - o de l'axe réel. Enfin, les deux autres éléments $M_{1} N$ et $N^{\prime} M_{1}^{\prime}$ donnent chacun un arc de circonférence joignant à $B$ un point de l'axe imaginaire.

Dans ces conditions, $A$ et $B$ étant positifs, il faut et il suffit, pour que le régime soit stable, que la courbe de réponse rencontre l'axe réel en un point d'abscisse inférieure à I pour une valeur de $\varphi$ inférieure à $\pi / 2$

Si A était négatif, et $B$ positif, la courbe pourrait se déduire de celle que nous venons d'étudier, par une symétrie autour de l'axe imaginaire. Elle entourerait donc le point $A$ un nombre infini de fois, et ceci, non du fait des pôles, mais du fait des racines: le régime serait donc instable.

Ce que nous avons dit du cas où $B$ serait négatif, $A$ restant positif, suffit à montrer que cette hypothèse est pareillement incompatible avec la stabilité.

Enfin, si nous supposons négatifs les deux paramètres $A$ et $B$, la fonction $R$ a deux pôles dans le demi-plan positif, pôles fournis par les zéros de $Y$. La stabilité exige alors que le point $A(1,0)$ soit entouré deux fois dans le sens direct, tandis que la courbe de réponse, ayant la disposition de la figure 26 , ne peut que l'éviter ou l'entourer dans le sens rétrograde.

Au total, la stabilité n'est possible que si $A$ et $B$ sont positifs, et sous la condition déjà indiquée pour ce cas. Explicitons donc la condition. Lorsque la courbe passe par $A$, on a en ce point :

$$
2 \rho \operatorname{tg} \varphi=\frac{A \varphi+\left(\varphi^{2}-B\right) i}{\frac{\varphi^{2}}{2}+B+A \varphi i}
$$

et par suite :

$$
\rho \operatorname{tg} \varphi=\frac{A \varphi}{\varphi^{2}+2 B}=\frac{\varphi^{2}-B}{2 A \varphi}
$$

système d'équations qui, mis sous la forme:

$$
A=\frac{B+\varphi^{2}}{2}-\frac{B}{\varphi^{2}}
$$

$$
\rho=\frac{1-\frac{B}{\varphi^{2}}}{2 A \frac{\dot{+g} \varphi}{\varphi}}
$$

conduit à construire le diagramme des régimes éoliens en haute chute donné à la figure 28 . On y a porté $A$ en ordonnées et $\rho$ en abscisses, puis tracé des courbes $B=$ Cte, et $\varphi=$ Cte. Par simple lecture, on déduit de la connaissance de deux quelconques des trois paramètres $A, B$, o la valeur que doit avoir. le troisième pour que le régime éolien se réalise; on lit en même temps la valeur de $\varphi=\frac{\omega L}{a}$ caractérisant la pulsation du mouvement entretenu qui en résulte. Lorsque les trois paramètres $A, B$, o sont donnés, on reconnaît que le régime est stable (insta. ble) à ce que, pour obtenir le régime éolien avec les valeurs de $A$ et $B$ envisagées, il faudrait que o soit plus élevé (faible) qu'il ne l'est: cette règle résulte immédiatement de la forme de la fonction $R_{0}$.

On remarquera que les valeurs admissibles pour $A$ et $B$ sont d'autant plus grandes que o est plus faible, sans toutefois croitre indéfiniment avec 1/o. Il faut cependant se garder d'en tirer la conclusion que la stabilité est compatible avec des réponses, tachymétrique $K_{0}$ et accélérométrique $K_{1}$, d'autant plus grandes que $\rho$ est plus faible, sans autre considération. Les paramètres $A$ et $B$ ne représentent pas exactement ces grandeurs, mais utilisent des facteurs $\frac{A}{K_{1}}=\frac{L}{\tau a}$ et $\frac{B}{K_{0}}=\frac{L^{2}}{\tau a^{2}}$ qu'on ne peut regarder dans tous les cas comme entièrement indépendants de $\rho$; en particulier, si la varia. tion de $\rho$ provient de ce que l'on considère des chutes de hauteurs différentes ( $\rho$ varie alors à peu près comme l'inverse de la chute), L/a qui varie grosso modo comme la chute est modifié en première approximation comme $1 / 0$ et cette modification contrarie les conséquences de l'autre.

Une amélioration des conditions de la comparaison peut être obtenue dans la mesure où le temps $\theta=\frac{2 \rho L}{a}$ peut être regardé comme constant d'une chute à l'autre; c'est là en pratique une approximation moins lointaine que celle qui regarderait $L / a$ comme invariable. Les expressions $A^{\prime}=2 \rho A=\frac{K_{r} \theta}{T}$ 




Fig. 28. - Régimes éoliens en hautes chutes.

N.-B. - Dans le texte, le nombre $\varphi$ mesure un angle en radians; c'est la mesure du même angle en degrés qui est notée $\Phi$ sur cette figure.

$$
\text { et } B^{1}=4 \quad \rho^{2} B=\frac{K_{0} \theta^{2}}{\tau}
$$

représentent alors convenablement $K_{\text {, et }} K_{0}$. $\mathrm{Ce}$ sont les expressions que nous avons déjà utilisées au chapitre précédent, notamment pour dessiner sur la figure 20 la limite de stabilité en basse chute : l'intérêt alors était précisément de montrer la dépendance de $K_{1}$ et $K_{0}$ à l'endroit de $\theta$. En les reprenant sur la figure 29, nous sommes conduits à tracer pour chaque valeur de - une courbe analogue à celle de la figure 20 . Chacune de ces courbes montre encore la dépen. dance de $K_{1}$ et $K_{0}$ par rapport à $\theta$, donc par rapport à l'inertie de l'eau. Quant aux comparaisons des diverses courbes entre elles, elles ne permettent des conclusions immédiates qu'à valeur constante de $\theta$. L'aspect de la figure ne doit donc pas faire conclure que la régulation est d'autant plus difficile et plus lente que o est olus faible : ceci ne serait vrai qu'à inertie égale; en d'autres termes, il convient de distinguer entre deux effets: I'un que I'on pourrait appeler d'inertie pure, est seul sensible en basse chute; l'autre, attribuable à l'élasticité, se présente comme une aggravation du premier en hautes chutes.

Ainsi, de deux installations ayant même temps $\theta$ caractéristique de l'inertie de l'eau, celle qui aura la conduite la plus courte, donc la plus grande valeur de $\rho$, admettra de plus grandes valeurs de $\frac{K_{0}}{\tau}$ et $\frac{K_{1}}{\tau}$. Mais, inversement, de deux installations alimentées par des conduites de même longueur, celle qui admettra les valeurs de $\frac{K_{0}}{\tau}$ et $\frac{K_{1}}{\tau}$ les plus élevées, sera celle que caractérise un paramètre $\rho$ plus faible: c'est qu'elle aura du même coup un temps $\theta$ plus faible, et qu'à longueur de canalisation constante $\left(L=\frac{a \theta}{2 o}\right)$ l'influence de $\theta$ 
l'emporte sur celle de o; la figure 28 a l'avantage de le montrer immédiatement.

De la dernière comparaison, on conclut en particulier :

1) une installation donnée, stable à une certaine puissance, l'est à toutes les puissances moindres (longueur constante: $\rho$ et $\theta$ diminuent ensemble).

2) Même en hautes chutes, ce n'est pas en élevant $\rho$, mais en l'abaissant que l'on contribue à rétablir la stabilité, parce que l'on modifie en même temps la vitesse $V$ et le temps $\theta$. II reste vrai cependant que, du fait des jeux contraires de o et de $\theta$, les variations de la vitesse $V$ influent moins sur la stabilité dans ces hautes chutes que dans les basses.

On comprendra maintenant plus facilement comment il se fait que l'on éprouve des difficultés de régulation aux deux extrémités de la gamme: hautes et basses chutes, mais non au milieu. C'est que :

1) en basses chutes, on rencontre des inerties élevées $\theta$.

2) en chutes moyennes, l'inertie réduite élimine les difficultés.

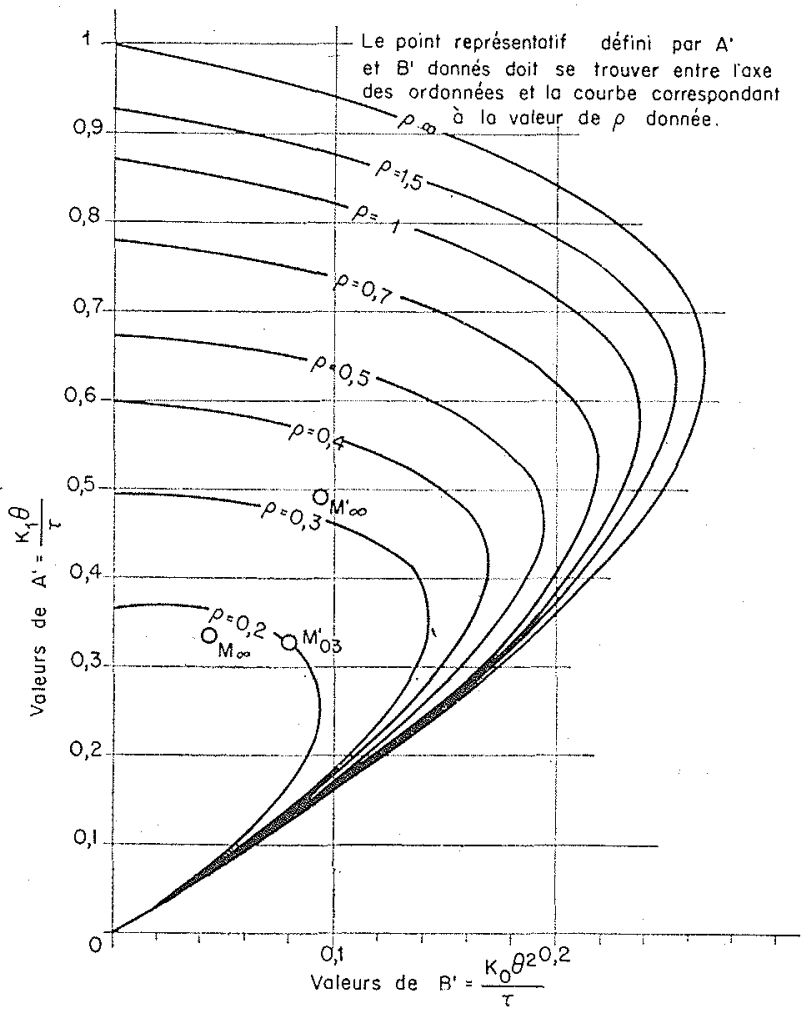

Fig. 29. - Condition de stabilité pour diverses valeurs du paramètre o d'Alliévi.
3) en hautes chutes, la longueur de la conduite les ramène sans que l'inertie prenne une valeur égale à celle que l'on rencontre en basses chutes.

Remarque: Bien que la figure 29 se déduise de la figure 28 par les correspondances: $A^{\prime}=20 A$ et $B^{\prime}=4 \quad 0^{2} B$, nous avons préféré adopter la même disposition pour la fig. 29 que pour lo fig. 20, afin de faciliter les comparaisons. Il est d'ailleurs possible d'en tracer les courbes par un calcul direct. Le système d'équations paramétriques (34) (35) se transcrit alors:

$$
\begin{aligned}
& A^{\prime}=2 A \rho=\frac{6 \rho^{2} \varphi \operatorname{tg} \varphi}{1+4 \rho^{2} \operatorname{tg}^{2} \varphi} \\
& B^{\prime}=4 \rho^{2} B=4 \rho^{2} \varphi^{2} \frac{1-2 \rho^{2} \operatorname{tg}^{2} \varphi}{1+4 \rho^{2} \operatorname{tg}^{2} \varphi}
\end{aligned}
$$

\section{Remarque concernant les limites de la zone de stabilité}

En hautes chutes, comme en basses chutes, la zone de stabilité est (pour chaque valeur de $o$ ) limitée à gauche par la droite $B=0$ et ailleurs par une courbe. L'établissement de la courbe montre que, de ce côté, et seulement de ce côté, la stabilité est limitée par un régime éolien correspondant à l'existence d'une racine purement imaginaire. On en conclut que la limite $B=O$ répond à l'existence $d^{\prime}$ une racine nulle...

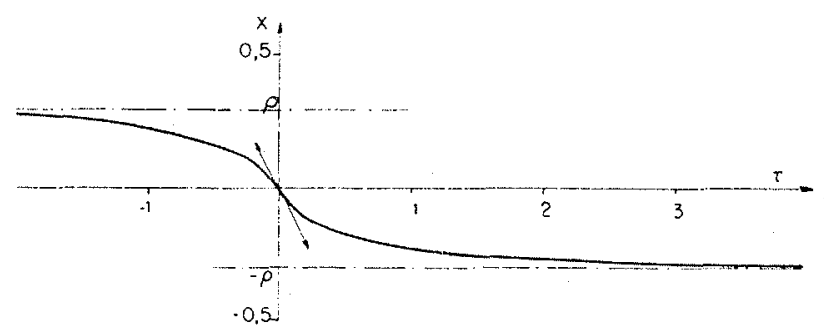

Fig. 30. - Variation de la fonction $X=\rho$ thr

11 est intéressant de le mettre directement en évidence.

Traçons les courbes représentatives des variations des fonctions $X$ et $Y$ de la variable $r$ limitée à ses valeurs réelles:

$$
X=-\rho \text { th } r \text { et } Y=\frac{r^{2}+A r+B}{r^{2}-2 A r-2 B}
$$

Tout point commun à ces deux courbes aura pour abscisse une racine réelle de la résolvante (18); il faut donc qu'il n'y en ait aucun du côté des obscisses positives

La fonction $X$ est représentée sur la figure 30 ; elle est de signe contraire à $r$, continuellement décroissante de $\rho$, lorsque $r$ est infiniment 
grand négatif, à - $\rho$, lorsque $r$ est infiniment grand positif. Elle passe à l'origine où elle présente un point d'inflexion dont la tangente a la pente $-\rho$.

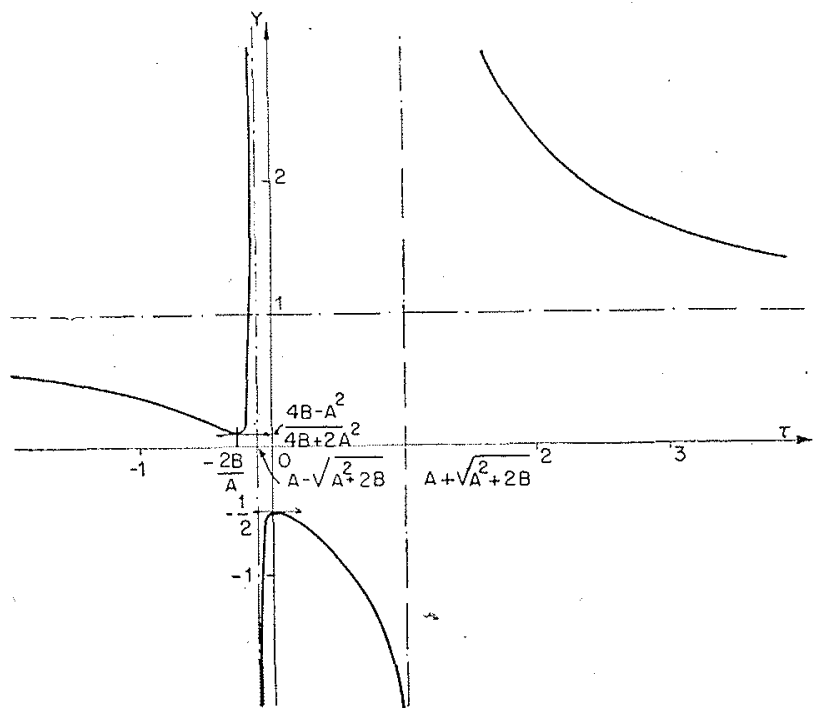

Fig. 31. - Variation de la fonction

$$
Y=\frac{r^{2}+A r+B}{r^{2}-2 A r-2 B}
$$

(cos de figure où $A$ et $B$ sont positifs)

La fonction $Y$ est représentée sur la fig. 31 , dans le cas normal où $A$ et $B$ sont positifs. Elle admet les asymptotes: $Y=1$ et $X=A \pm$ $\sqrt{A^{2}+2 B}$. Elle présente un maximum $-1 / 2$ pour $r=0$ et un minimum $\frac{4 B-A^{2}}{4 B+2 A^{2}}$ pour $r=-\frac{2 B}{A}$. Si $B$ devenait négatif, la courbe prendrait la disposition de la figure 32.

Dans les deux cas de figure, les racines réelles, s'il en existe, correspondent à des points de lo branche de gauche ou de la branche intermédiaire. Le cas où $B$ est négatif apparaît immédiatement instable au simple examen des figures 30 et 32 ; les deux courbes ont nécessairement un point commun entre l'axe des ordonnées et la première asymptote verticale.

Lorsque $B$ est positif (figure 31), la première branche est tout entière du côté des $r$ négatifs; nous n'avons donc pas à craindre "d'instabilité du fait d'une intersection de ce côté. Par ailleurs, lorsque les conditions de stabilité exprimées par la figure 29 sont remplies, la courbe $Y$ n'a du côté des $r$ positifs, aucun point commun avec la tangente à l'origine à la courbe $X$. En effet, remplacer la courbe $X$ par sa tangente à l'origine, revient à étudier le mouvement en masse, et par suite, le fait que cette tangente rencontre la courbe $Y$ du côté des $r$ positifs impliquerait un mouvement en masse instable: or, d'après la figure 29, une telle instabilité est impossible dans notre hypothèse; par suite, la droite envisagée ne rencontre pas la courbe $Y$ à droite de l'axe des ordonnées. A fortiori, la courbe $X$ ne la -ncontre-t-elle pas. Le changement de signe de b entraine donc bien celui d'une racine réelle.

\section{Limites et Méthodes de l'étude quantitative}

Le paragraphe précédent nous a montré une double différence entre les fonctionnements des installations de haute et de basse chute :

1) en haute chute, l'influence de l'élasticité s'ajoute à celle de l'inertie, et rend plus dures les conditions de stabilité (figure 29).

2) En haute chute, la période du mouvement auto-entretenu est toujours supérieure à $4 \mathrm{~L} / \mathrm{a}$, période propre de la conduite. Nous devons donc nous attendre à irouver un délai de réglage accru par les phénomènes dus à l'élasticité si du moins, la notion même de délai garde un intérêt. Il est permis de se demander en effet si les oscillations de la conduite n'auront pas une influence prépondérante: le début du mouvement de réglage produirait une onde de pression dans la conduite, et cette onde revenant, changée de signe, au bout $d^{\prime} u n e$ demi-période, provoquerait un mouvement supplémentaire, lui-même suivi d'un autre après un temps équivalent... et ainsi de suite sans amortissement autre que celui dont les pertes de charge dans la conduite sont à l'origine. Nous montrerons qu'il n'en est rien, et qu'il existe



Fig. 32. - Variation de la fonction

$$
Y=\frac{r^{2}+A r+B}{r^{2}-2 A r-2 B}
$$


réellement un optimum analogue à celui que nous avons déterminé pour les basses chutes.

Toutefois, pour alléger la discussion, nous nous bornerons à étudier le cas où le paramèrre o d'ALLIEVI est égal à 0,3 . Nous pouvons nous le permettre sans diminuer sérieusement la portée de notre travail parce que :

1) Les difficultés de régulation que nous nous proposons d'élucider et même, autant que nous le pourrons, d'éliminer, se manifestent seulement dans les installations de basse et dans celles de haute chute, mais rarement dans celles de moyenne chute.

2) Tandis qu'au premier de ces cas, répondent les valeurs élevées de o et l'approximation du mouvement en masse qui a fait l'objet du chapitre précédent, au second correspondent les valeurs faibles entre lesquelles 0,3 représente une moyenne ou même approche du minimum pratique qu'offrent des chutes de 1.000 mètres par exemple.

3) L'étude faite pour $o=0,3$ pourrait au besoin se renouveler pour tout autre cas; les résultats généraux que nous acquerrons au cours du travail simplifieraient cette reprise; en outre, les valeurs numériques elles-mêmes constitueraient des indications de nature à limiter les tâtonnements.

Le principe de la méthode reste le mème qu'au chapitre précédent. Nous devons d'abord chercher à accroitre le plus possible le plus faible des amortissements indiqués par les parties réelles des racines de l'équation (18). Toutefois, il faut se rappeler qu'il s'agit d'une équation trans. cendante: le nombre des racines est illimité, mais toutes n'ont pas le même intérêt. Tout d'abord, au point de vue du calcul, il faut remarquer que ce qui concerne les pulsations élevées ne peut être retenu comme exact, parce que des phénomènes de même durée ont été négligés dans la mise en équations et notamment les oscil. lations propres du tachymètre et de l'accéléromètre: c'est en effet par une fiction, de valeur très réelle, mais purement pratique, que nous avons pu écrire nos équations comme si ces appareils indiquaient sans aucun retard, l'un l'écart de vitesse, l'autre l'accélération. Ensuite, au point de vue physique, on sait que toute fonction périodique est décomposable en une série de fonctions sinusoïdales; le fait de rencontrer une telle série pour solution d'un système d'équations différentielles ne doit donc pas induire à en étudier chacun des éléments comme une fonction sinusoidale distincte. Techniquement, on a coutume de désigner l'ensemble sous le nom de sinusoïde déformée; en première approximation, il est représenté par la sinusoïde de plus grande période, les amplitudes des autres composantes étant assez faibles devant la sienne pour pouvoir être assimilées à des corrections ou «déformations》.

Ici, une difficulté supplémentaire viendra des ce que les composantes ne sont pas de simples sinusoïdes, mais des produits de sinusoïdes par des exponentielles, ou suivant la terminologie technique, des sinusoïdes amorties. De ce fait, la plus basse fréquence pourra perdre de son importance au profit de celle qui lui est immédiatement supérieure; nous ne pourrons donc pas toujours nous borner à l'étude de l'oscillation fondamentale, mais nous devrons dans certain's cas, étudier le ou les premiers harmoniques; le fait se produira surtout au chapitre $V I I$ où nous étudierons un système de régulation rapide.

Nous aurions été heureux de donner ici comme au chapitre $V \S \S 7$ et 8 , des courbes de variation de vitesse au cours des petits mouvements de réglage, mais la valeur pratique de courbes «calculées » eût été bien minime. Du fait de la validité limitée de nos équations, les conclusions concernant les fréquences élevées eussent été trop approximatives; quant à celles concernant les plus basses, elles n'auraient pas ajouté grand' chose au reste de l'étude : étude du mouvement en masse et recherche du facteur d'amortissement.

Cette partie de notre travail ne portera donc que sur les racines de l'équation (18) et, à titre d'exemple, spécialement dans le cas où $o=0,3$. Elle utilisera les méthodes déjà esquissées:

1) tracé des courbes représentant les fonctions $X$ et $Y$ afin d'étudier les racines réelles.

2) tracé des courbes $R y$ analogues à la courbe de réponse $R_{11}$ mais obtenues en donnant à la variable $r$ de la fonction $R$ des séries de valeurs $r=-\gamma \mathrm{i}+\mathrm{i} \varphi$ (au lieu de $r=i \varphi$ ) $v$ étant une constante pour chacune des courbes $\mathrm{R} \gamma$.

\section{Etude des racines réelles}

Reprenons les courbes représentatives des fonctions $X$ et $Y$ lorsque la variable $r$ est réelle (Figures 30 et 31 ). Les abscisses de leurs points communs, $s^{\prime} i l$ en existe, indiquent les valeurs de $\gamma=\frac{\lambda L}{a}$ caractérisant les amor-

tissements des composants apériodiques éventuels. Ces points, nous l'avons établi au paragraphe $4 \mathrm{ci}$-dessus, ne peuvent se trouver que su: la branche gauche de la courbe $Y$. Nous allons chercher comment il convient de modifier $A$ et $B$ pour éloigner le plus possible ces points da l'axe des $Y$. Puisqu'il s'agit avant tout du sens des modifications et de l'existence d'un optimum, 
plus que de sa valeur, nous pourrons assimiler th $r$ à $r$, donc à $\gamma$, d'autant que ces nombres sont faibles, $\varphi$ étant nul et $\gamma$ ne retenant notre attention que parce qu'il n'est pas très grand. L'équation (18) s'écrira donc:

\begin{tabular}{l}
$\rho \gamma^{3}+\left(1-2 A_{\rho}\right) \gamma^{2}+\left(2 B_{\rho}-A\right) y$ \\
\hline$B$
\end{tabular} et même, en négligeant $\gamma^{3}$, ce qui peut être accepté en première approximation, et toujours à raison du but limité que nous nous proposons pour l'instant :

$\left(1-2 A_{\rho}\right) \gamma^{2}-(A-2 B \rho) \gamma+B=0$

équation qui admet deux racines réelles ou imaginaires. Leurs parties réelles sont positives si le régime est stable: on voit en effet, d'après la figure 29, que $1-2 \mathrm{~A} O=1-\mathrm{A}^{\prime}$ est positif ainsi que $A-2 B \rho=\frac{A^{\prime}-B^{\prime}}{2 \rho}$

Ecartons pour l'instant le cas des racines imaginaires qui sera étudié plus loin, en remarquant cependant que la somme de ces premières racines (par laquelle est caractérisé l'amortissement du mouvement correspondant), $A$ et $o$ étant donnés, diminue lorsqu'on augmente $B$. Quant au cas où les racines sont réelles, il demande au contraire que pour augmenter l'amortissement, on augmente $B$ : c'est que, si la diminution de $B$ augmente bien la somme des racines (qu'elles soient réelles ou imaginaires), elle diminue leur produit, et par conséquent diminue la plus faible d'entre elles, ce qui seul nous importe ici. Aussi le maximum de l'amortissement le plus faible (racine réelle la plus faible, ou demi-somme des racines imaginaires) sera-t-il réalisé lorsque les deux racines seront égales, ou en d'autres termes, lorsque les courbes représentant $X$ et $Y$ seront tangentes, ceci sans préjudice de l'intérêt qu'il y aura en pratique à se placer dans le cas des racines imaginaires, malgré un amortissement légèrement amoindri : nous retrouvons les caractères connus de l'amortissement critique.

Jusqu'ici, nous avons supposé $A$ et $\rho$ donnés. En fait, $\rho$ est seul imposé. Nous devons donc chercher quelle modification de $A$ permet d'augmenter l'amortissement maximum que nous venons de déceler pour chaque valeur de A. Nous continuerons à utiliser l'équation du deuxième degré (37). La valeur de B correspondant à la racine double est définie par :

$$
(A-2 B \rho)=-4 B(1-2 A \rho)=0
$$$$
4 \mathrm{~B}^{2} \rho^{2}-4 \mathrm{~B}\left(1-\mathrm{A}_{\rho}\right)+\mathrm{A}^{2}=0
$$

dont la plus faible solution est seule à retenir, parce que, si B était compris entre les deux solutions, l'équation (37) aurait des racines imaginaires et I'on devrait réduire $B$ pour accroitre l'amortissement. On a done:

$$
\begin{aligned}
& B=\frac{1-A \rho-\sqrt{1-2 A \rho}}{2 \rho^{2}} \\
& \gamma=\frac{A-2 B \rho}{2(1-2 A \rho)}=\frac{1}{2 \rho}\left(\sqrt{\left.\frac{1}{1-2 A}-1\right)}\right.
\end{aligned}
$$

fonction croissante de A. Dans la limite de validité de nos hypothèses, nous avons done intérêt à augmenter A pour élever l'amortisse. ment apériodique; nous devons en même temps, choisir $B$ tel que les courbes $X$ et $Y$ soient tangentes; toutefois, il est intéressant en pratique de donner à $B$ une valeur un peu supérieure, de manière à substituer au mouvement apériodique un mouvement oscillant à faible fréquence.

\section{Définition et tracé de la courtbe $\mathbb{R} \gamma$ ef des courbes ouxiliaires $X_{\gamma}$ ef $Y_{\gamma}$}

Nous utiliserons au cours de notre étude, les fonctions complexes de $\varphi$ :

$$
\begin{aligned}
& x_{\gamma}=-\rho^{\text {th }}(\varphi \mathrm{i}-\gamma) \\
& \text { et } \quad Y_{\gamma}=\frac{(\varphi i-\gamma)^{2}+A(\varphi i-\gamma)+B}{(\varphi i-\gamma)=2 A(\varphi i-\gamma)-2 B}
\end{aligned}
$$

La première peut se mettre sous la forme:

$$
x y=o \frac{\operatorname{sh} 2 \gamma-i \sin 2 \varphi}{\operatorname{ch} 2 \gamma+\cos 2}
$$

Elle est représentée dans le plan complexe. por une circonférence dont le centre est sur l'axe réel au point d'abscisse $\frac{\rho}{\text { th } 2 y}$; son rayon est : $\frac{\rho}{\operatorname{sh} 2 \gamma}$. Elle rencontre l'axe réel au point d'abscisse $\rho$ th $\gamma$ pour chaque valeur de $\varphi$ multiple de $\pi$ et au point $\frac{\rho}{\text { th } \gamma}$ pour les valeurs de $\varphi$ multiples impairs de $\frac{\pi}{2}$. Elle est parcourue dans le sens direct lorsque $\varphi$ augmente; on peut la coter en valeurs de $\varphi$ en remarquant que l'argument a pour tangente : $\frac{-\sin 2 \varphi}{\operatorname{sh} 2 \gamma}$; on notera, ainsi que le montre la figure 33, que les graduations s'espacent d'autant plus que $\varphi$ est plus voisin $d^{\prime} u n$ multiple impair de $\frac{\pi}{2}$, et 
se resserrent au contraire davantage au voisinage de $k \pi$; le fait est d'autant plus marqué que $\gamma$ est plus faible.

Les diverses circonférences $X Y$ admettent pour centre radical, l'origine, dont la puissance par rapport à chacune d'elles est $\rho^{2}$. Chacune est intérieure à toutes celles qui correspondent à une valeur plus faible mais positive de y. A la limite, $y$ s'annulant, la circonférence se confond avec l'axe imaginaire: $X_{0}=-i \operatorname{tg} \varphi$ forme que nous avons utilisée dans l'étude quaiitative.

La deuxième fonction s'écrit :

$$
Y \cdot \gamma=\frac{\gamma^{2}-\varphi^{2}-A \gamma+B+(A-2 \gamma) \varphi i}{\gamma^{2}-\varphi^{2}+2 A \gamma-2 B-2(A+\gamma) \varphi i}
$$

et présente des caractères analogues à ceux de la fonction $Y_{1}$ étudiée ci-dessus au paragraphe 1 : en particulier, elle n'est pas périodique; la courbe représentative est parcourue d'un mouvement continu. Elle part avec $\varphi=0$ du point de l'axe réel ayant pour abscisse $\gamma^{2}-A \gamma+B$

$\gamma^{2}+2 A y-2 B$ et aboutit lorsque $\varphi$ croit indéfiniment au point $A(I, O)$ ainsi qu'on $I^{\prime} a$ indiqué sur la figure 33 pour le même cas particulier qui a fait l'objet des figures 29 et 30 . On notera qu'aux valeurs élevées de $\varphi$, les points représentatifs restent très proches de $A$ $(1, \mathrm{O})$.

Des deux tracés des courbes $X X_{y}$ et $Y Y$, on déduit facilement celui de $R_{\gamma}$ qui a été représenté sur la figure 34. La fonction $R y$ est en effet le quotient des deux précédentes $\frac{X_{Y}}{Y_{Y}}$ l'argument d'un point coté $\varphi$ sur cette courbe est la différence des arguments des points de même cote des deux courbes auxiliaires; le module est le rapport des deux modules. Dans le cas particulier auquel répond la figure 34, la courbe part du voisinage de $\mathrm{A}$ lorsque $\varphi=0$, le laissant à gauche, tourne autour de lui, revient dans son voisinage lorsque $\varphi$ atteint $80^{\circ}$ environ, revient après un nouveau tour lorsque $\varphi$ approche de $\frac{3 \pi}{2}$, puis lorsqu'il approche de $5 \pi$ $\frac{5 \pi}{2}$... La courbe tend asymptotiquement vers la circonférence $x_{y}$, entourant continuellement le point A qu'elle laisse toujours à gauche. On en conclut que les racines ont toutes une partie réelle dont la valeur absolue est supérieure à 0,25 , valeur de y adoptée pour tracer la courbe. Qui voudrait achever le calcul des racines le pourrait facilement en utilisant les remarques faites au chapitre $11, \S \S 7$ et 8 . II tracerait au voisinage de la courbe, et pour chacun de ses passages près de $A$, un quadrillage représentant les lignes $\gamma=$ Cte et $\varphi=$ Cte la dimension des mailles étant indiquée par la graduation de $\mathrm{R} y$ en valeurs de $\varphi$; la position de $\mathrm{A}$ dans ce quadrillage indiquerait la valeur de $\varphi$ pour chaque racine et l'excédent de la valeur corres. pondante de $y$ sur la valeur de base, ici 0,25. II suffirait d'ailleurs de le faire pour les premières racines, d'abord parce que c'est seulement

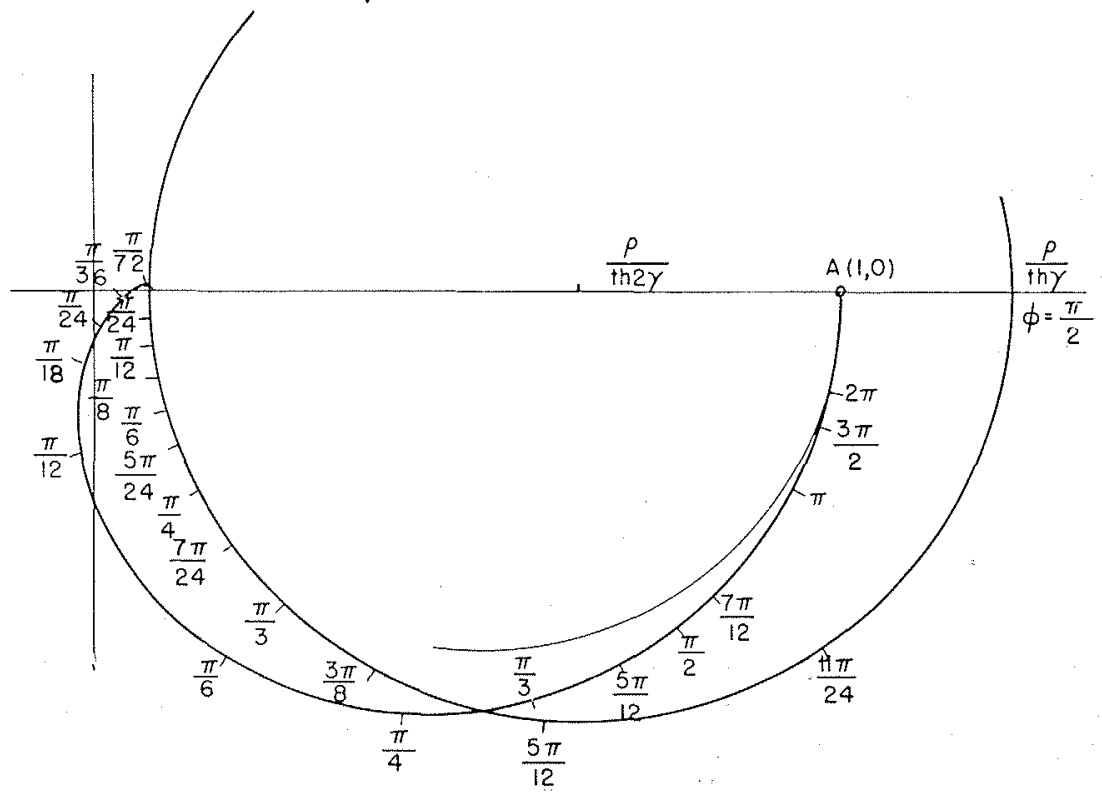

Fig. 33. - Circonférence représentant les variations périodiques de $X_{Y}$ et courbe indiquant les valeurs de $Y_{Y}$ IOn a pris à titre d'exemple $o=0,3 ; A=0,44 ; B=0,06$ et $\gamma=0,25$ ). 
pour elles que l'étude est valable, ensuite parce que, de toute évidence, lorsque $\varphi$ s'élève, $\gamma$ tend vers $o$

On notera cependant que la méthode d'évaluation graphique signalée doit être regardée plutôt comme une extrapolation que comme une interpolation, et qu'il convient par conséquent, de la manier avec beaucoup de prudence. En particulier, on reconnaitra au seul aspect des figures 26 et 34 établies cependant pour les mêmes valeurs de $A, B$, et $o$, le caractère illusoire qu'aurait une appréciation des racines, partie imaginaire ou partie réelle, en utilisant seulement la courbe de réponse $R_{0}$.

\section{$7^{\circ}$ Etude des racines complexes}

II n'est pas nécessaire pour étudier les racines complexes, de dessiner la courbe $R_{\gamma}$ : les courbes auxiliaires $X Y$ et $Y \gamma$ peuvent en tenir lieu. A tout passage de $R^{u} Y$ au point $A$, correspond en effet un point commun à deux courbes $X_{Y}$ e $Y_{Y}$ de même indice $\gamma$ et ayant même cote $\varphi$ sur les deux courbes. L'emploi de ces courbes auxiliaires constitue une méthode pratique d'étude des racines en raison des séparations relativement nettes qu'elle introduit entre les influences des divers éléments en cause. Nous avons vu ci-dessus $(\$ 6)$ que les courbes $X y$ ne dépendent que de $\gamma$, $\rho$ étant pour toute l'étude une constante. Au contraire, sauf pour les valeurs faibles de $\varphi$, que d'ailleurs nous avons envisagées précédemment en partie (au $\$ 5$ ), les courbes $Y$ Y dépendent beaucoup moins de $\gamma$, mais traduisent toute l'influence de $A$ et de $B$. Enfin, il est intéressant de noter que l'échelle des $\varphi$ sur $X_{y}$ présente un caractère périodique que n'a pas l'échelle sur $Y_{Y}$ : l'influence de $Y$ sur la valeur de la fonction $R \gamma$, comme celle de $\gamma$ se traduit en majeure partie par une variation de la fonction $X_{\gamma}$.

Aussi, en pratique, la recherche des racines lorsque $A$ et $B$ sont donnés, consiste-t-elle à tracer le réseau des circonférences $X_{Y}$, cotées en valeurs de $\varphi$, puis à disposer sur ce canevas, le faisceau correspondant des courbes $Y_{Y}$, ellesmêmes cotées en valeurs de $\varphi$; les points communs apparaissent très vite. On peut au besoin achever le calcul par une interpolation faite en recourant à des courbes $R y$ tracées à grande échelle, et seulement au voisinage immédiat des racines détectées par ce premier travail.

Nous ne nous livrerons pas ici à ce calcul, car nos indications numériques n'étant prises qu'à titre d'exemple, la figure 34 nous fixe avec une précision amplement suffisante sur la valeur des racines. Par contre, il est intéressant de montrer certains caractères de ces racines à cause de leur généralité. Lorsque nous faisons croître $\varphi$ à partir de zéro, nous rencontrons d'abord deux racines, soit réelles, soit imaginaires, mais à faible valeur de $\varphi$. Réelles, elles ont été étudiées au $\$ 5$; imaginaires, elles se déterminent facilement parce qu'aux faibles valeurs de $\varphi$, la graduation de $X Y$ est très serrée et celle de $Y$ y lâche. Nous désignerons éventuellement le mouvement correspondant par le qualificatif: «QUASI APERIODIQUE ». Au delà, nous trouvons une autre racine (un autre couple de raci. nes) imaginaire pour laquelle la valeur de $\varphi$ reste inférieure à $\pi / 2$ : nous appellerons le mouvement correspondant « OSCILLATION FONDAMENTALE » en raison de sa périodicité qui appròche de celle de la conduite: $4 \mathrm{~L} / \mathrm{a}$, et parce que les oscillations d'ordre supérieur en sont sensiblement les harmoniques impairs : 3,5 .. Chacune de ces racines répond en effet à une valeur de $\varphi$ supérieure de plus de $\pi$ à la précédente, mais restant inférieure la première à $3 \pi / 2$, la seconde à $5 \pi / 2 \ldots$ Ce fait ressort immédiatement de la comparaison des courbes $X \gamma_{\text {et }} Y_{\gamma}$ dans la région des valeurs élevées de $\varphi$. La disposition relative de celles-ci est en effet toujours semblable à celle de la fig. 33, car aux valeurs élevées de $\varphi$, la courbe $Y \gamma$ tend vers la circonférence $C$ tracée sur cette figure et définie par l'expression :

$$
Y^{\prime} \gamma=\frac{\varphi \mathrm{i}+\mathrm{A}-2 \cdot \gamma}{\varphi \mathrm{i}-2(\mathrm{~A}+\gamma)}
$$

obtenue en négligeant dans $Y$ Y les termes de degré nul en $\varphi$.

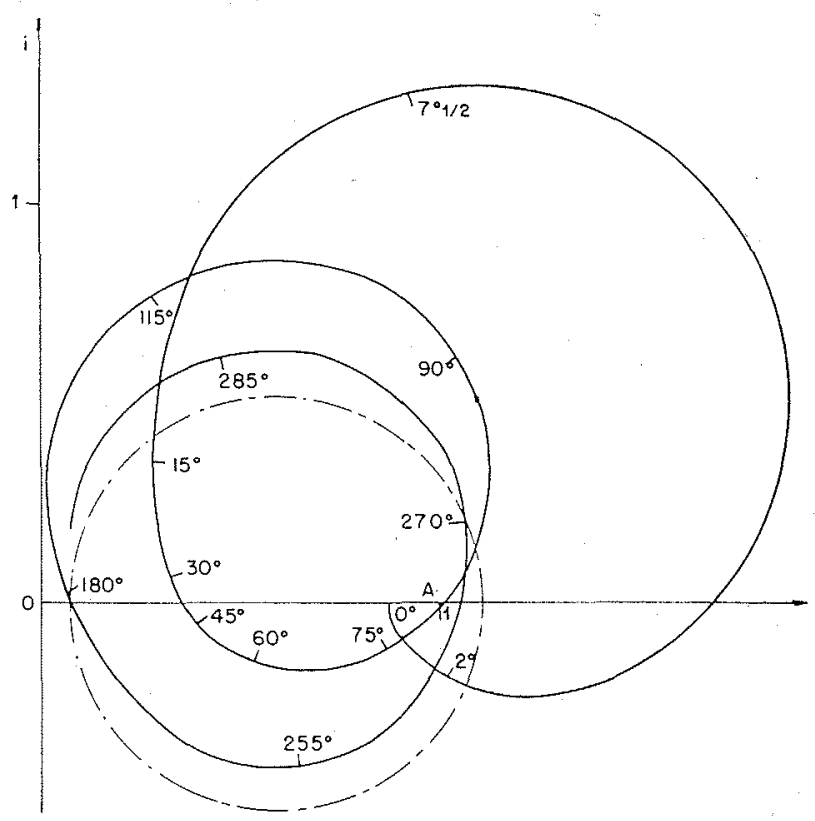

Fig. 34. - Exemple de courbe $R y$. On a adopté $o=0,3$ $A=0,44 ; B=0,06$ 
Pour la même raison, l'amortissement des harmoniques élevés est toujours voisin de $\lambda=\frac{\rho \text { a }}{L}$ tel que $y=o$. La courbe $X_{y}$ passant par le point $A(I, O)$ ou aboutissent toujours $C$ et $Y$.

\section{$8^{\circ}$ Combinaison optimum}

La recherche de la combinaison A, B la meilleure doit être conduite de manière à obtenir l'amortissement rapide du mouvement quasiapériodique et de l'oscillation fondamentale, les oscillations d'ordre supérieur n'ayant, avonsnous dit, qu'une importance limitée dans l'état actuel des choses. Nous venons d'ailleurs de voir qu'elles ne sont pas susceptibles de modifications sérieuses de leur amortissement par variations de $A$ ou $B$.

En étudiant le mouvement quasi-apériodique au paragraphe 5 ci-dessus, nous avons trouvé qu'à chaque valeur de $A$, répond une valeur de $B$ réalisant l'amortissement critique, et au-dessus de laquelle il convient de se tenir pour qu'une oscillation à faible pulsation rende plus rapide le retour à l'équilibre. Nous avons reconnu en outre, qu'on augmente cette valeur de $B$ et I'amortissement quasi-apériodique en augmentant A.

Pour augmenter l'amortissement de l'oscilla tion fondamentale, on doit « rabattre » la courbe Y y vers l'axe réel, donc diminuer A. II est cependant inutile de pousser trop loin dans cette voie pour deux raisons. D'une part, on diminue-

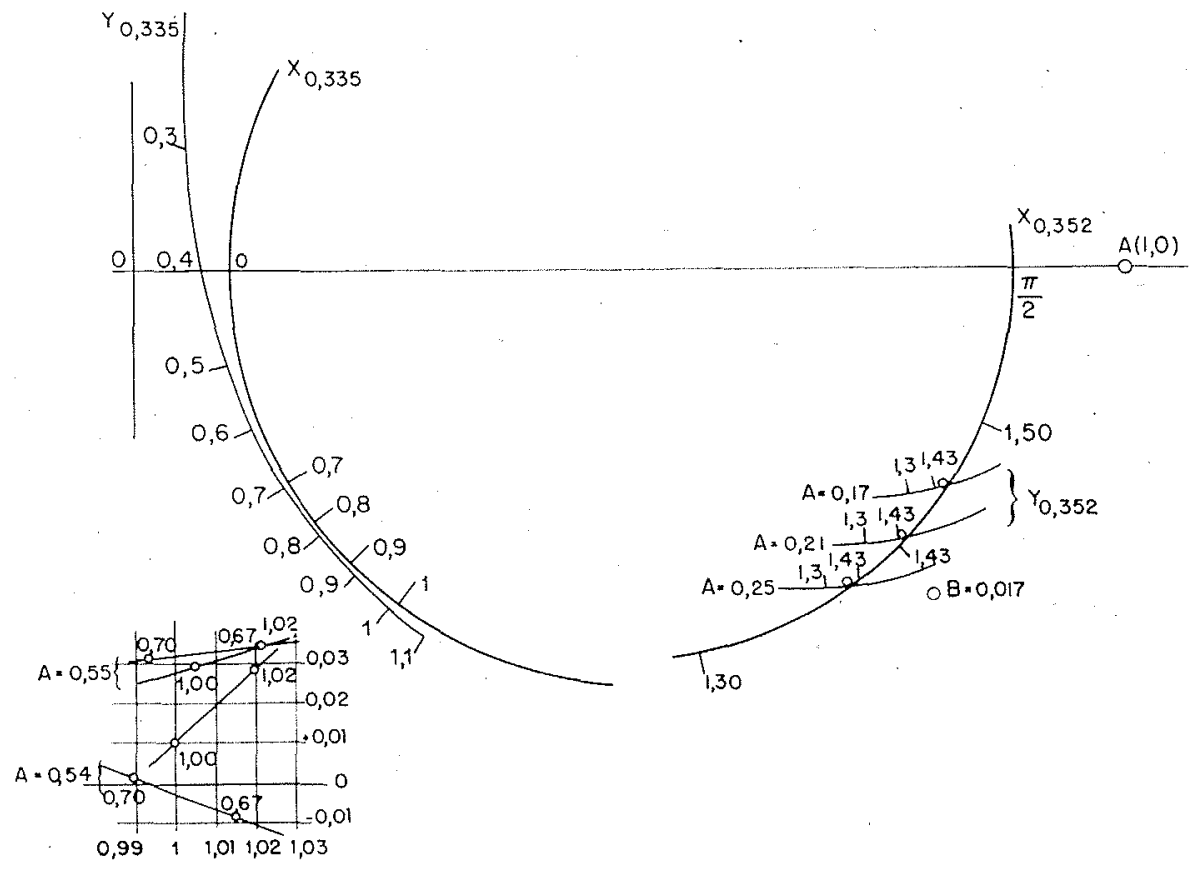

Fig. 35. - Etude du dimensionnement optimum d'un régulat eur de vitesse en haute chute $(\rho=0,3)$. rait trop l'amoritissement quasi - apériodique $D^{\prime}$ autre part, la diminution de $A$, si elle rapproche bien les points de $Y_{\gamma}$ de l'axe réel, ne les déplace pas nécessairement vers des courbes $X_{Y}$ à indice plus élevé. Dans le cas où $\rho=0.3$ et où $B=0,075$, le minimum utile de $A$ est approximativement 0,21 auquel correspond ia racine $\varphi=1,43$ et $\gamma=0,352$, maximum pour cette valeur de $B$, ainsi que nous allons le montrer. Traçons sur la figure 35 (partie droite de la figure):

1) la circonférence $X_{0,352}$ avec les quatre points pris à titre de repères: $\varphi=\pi / 2,1,5$, 1,43 et 1,3 .

2) les trois courbes $Y_{0,352}$ pour $B=0,075$, mais répondant aux valeurs de $A$ indiquées près de chacune: $0,17,0,21$ et 0,25 ; sur toutes trois, repérons les points $\varphi=1,3$ et $\varphi=1,43$.

A partir de ces éléments, nous pouvons marquer sur chacune des courbes $Y_{0,35}$ le point qui a même cote $\varphi$ que le point voisin sur la courbe $X_{0,252}$ et auquel correspond par conséquent une intersection de la coưrbe $R_{0,352}$ avec l'axe réel: sur la figure, nous avons repéré ces trois points par un petit cercle. On peut observer que dans le cas de figure envisagé, les trois points sont pratiquement sur la circonférence $X_{0,352}$, de telle sorte qu'avec la même approximation les trois courbes $R_{0,352}$ correspondantes passent en $A$ $(0,1)$. C'est-à-dire que dans les trois cas, nous sommes en présence d'une racine de la résolvante; la partie réelle $\gamma \neq 0,352$ n'est pas modifiée par la variation de A. Cette valeur de $\gamma$ 
est donc bien un maximum relativement aux variations de $\mathrm{A}$. Comme nous le disions à l'instant, le point figuratif de la racine sur la courbe $Y_{n .352}$ (point cerclé), bien que se déplaçant vers l'axe réel lorsque $A$ diminue, cesse de se déplacer vers des courbes $X y$ à indice plus faible, sa trajectoire étant tangente à l'une de ces courbes.

Notons que ce maximum dépend de $B$ et diminue avec lui ; nous avons figuré, en le repé. rant, $B=0,017$, un point de la courbe $Y_{0,352}$ répondant à cette valeur de $B$ et à $A=0,21$ (11 a pour cote $\varphi=1,43)$; on voit immédiate. ment d'après sa position sur le canevas des courbes $X$ y que l'amortissement de l'oscillation fondamentale avec $B=0,017$ serait nettement inférieur à celui qui est obtenu avec $\mathrm{B}=0,075$.

On peut se demander si en hautes chutes, où $\rho$ est particulièrement faible, cet amortissement maximum de l'oscillation fondamentale ne reste pas inférieur à l'amortissement quasi-apériodique autorisé par la même valeur de $A$; il représenterait alors le véritable optimum. En fait, pour $o=0,3$, cette éventualité ne se réalise pas, car l'amortissement apériodique maximum outorisé par $A=0,21$ correspond sensiblement à $y=0,12$ très inférieur, non seulement au maximum obtenu en supposant $B=0,075$, mais aussi à celui que l'on obtiendrait en utilisant la valeur 0,017 de $B$ qui, précisément permet d'atteindre le maximum $y=0,12$ pour la valeur de A considérée: 0,21 . L'optimum répond donc à des valeurs de $A$ et $B$ supérieures à celles-ci.

Augmentant progressivement $A$, et déterminant chaque fois $B$ pour que le mouvement quasi-apériodique ait une pulsation double de son amortissement, nous parviendrons à une combinaison pour laquelle les deux composantes étudiées auront même amortissement. Cette combinaison constituera une bonne approxima.tion de l'optimum pratique. Sans doute, préférera-t-on s'en tenir à une valeur de $A$ un peu moindre, de façon à amortir davantage l'oscillation fondamentale? Pour pouvoir le discuter, il faudrait, soit tracer la courbe de variation de la vitesse au cours des petits mouvements de réglage, soit, mieux, se livrer à une étude expérimentale. De toute manière, le gain serait peu important et difficile à chiffrer. La combinaison indiquée peut donc à juste titre être regardée comme un optimum pratique.

Nous avons trouvé pour le cas étudié $(\rho=0,3)$ que $A$ devait être voisin de 0,54 et $B$ de 0,225 . On obtient alors $\gamma \neq 0,333$ avec $\varphi_{0}=0,69$, ef $\gamma \neq 0,330$ avec $\varphi_{1}=0,995$. La partie gauche de la figure 35 donne les dernières courbes $X_{0,333}$ et $Y_{0,135}$ qui ont été utilisées dans la recherche; nous $y$ avions adopté: $A=0,55, B=0,225$ (et $\gamma=0,335)$. A côté de ce tracé, nous donnons aussi à grande échelle, les portions utiles de courbes $R_{0,335}$, l'une pour $A=0,55$, $B=0,225$, l'autre pour $A=0,54, B=0,225$; les valeurs des racines y apparaissent immédiatement.

Remarque I. - Nous n'avons pas eu jusqu'ici la possibilité de vérifier expérimentalement ces résultats qui sortent du cadre des travaux de laboratoire. Nous leur trouvons pourtant un appui sérieux, d'origine pratique, dans l'ouvrage de $M$. GADEN. Cet auteur donne 1,67 pour valeur moyenne du rapport $x$ de la période des oscillations à la période propre de la conduite dans les installations qui donnent satisfaction, et où $o$ est de 0,3 . Or, à ce rapport $x=1,67$, répondrait un angle $\varphi=\frac{\pi}{2 x}=0,94$, compris entre $\varphi_{1}$ et $\varphi_{1}$ et voisin de $\varphi_{1}$

Remarque II. - Nous avons repéré sur la figure 29, par l'indication $M_{0,3}^{\prime}$ l'optimum pratique que nous venons de calculer pour la valeur 0,3 de $o$. Nous y avons signalé aussi l'optimum pratique dans I'hypothèse du mouvement en masse par le symbole $M^{\prime} \infty$ et l'optimum théorique correspondant par $M$. La position de chacun de ces points par rapport à la courbe limite de stabilité répondant à la même valeur de $\rho$ est intéressante à noter. On observera en particulier que $M^{\prime} \infty$ et $M^{\prime}{ }_{0,3}$ occupent des positions assez semblables; la similitude eût été plus accentuée si nous avions admis en basse chute un décrément un peu moindre (Répondant par

exemple à $n=\frac{\omega}{i}=3$ au lieu de $n=21$,

ou si, en haute chute, nous avions accordé davantage d'importance à l'oscillation fondamentale, et en conséquence, diminué légèrement $A$. La similitude ainsi reconnue entre les cas extrêmes doit selon toute vroisemblance, se réaliser aussi entre eux et les cas intermédiaires. Elle constitue à tout le moins, une première approximation que I'on pourrait vérifier pour chaque problème particulier, et à laquelle on apporterait éventuellement les corrections nécessaires. Nous n'étudierons pas davantage ces cas moyens, d'autant qu'en pratique, ils ont rarement donné lieu aux phénomènes de pompage auxquels nous cherchons à porter un remède. 


\section{CHAPITRE VII}

\section{REMEDE AUX EFFETS DUS A L'INERTIE DE L'EAU}

\section{1'Rappel des élémentrs du probième ef des solutions classiques}

Nous avons exposé au début de ce travail, les difficultés auxquelles se heurte le réglage auto. matique rapide de la vitesse des turbines hydrauliques. L'inertie de l'eau s'oppose aux variations de débit, et plus encore de puissance, que tend à produire le régulateur au cours des manceuvres de réglage; elle le fait d'autant plus qu'il manœuvre plus vite. De ce fait, elle est d̀ l'origine de surréglages dont l'importance est parfois telle qu'elle empêche l'amortissement des oscillations de réglage. Le phénomène est connu sous le nom de pompage. Il se manifeste dans les installations de basse chute parce que le débit élevé de l'eau y rend l'inertie importante; et également, malgré une inertie souvent moindre, dans les installations de haute chute où les phénomènes d'élasticité accentuent les effets de I'inertie.

Nous avons montré que l'on peut concrétiser cette double influence de l'inertie et de l'élasticité de l'eau sur la régulation par un « délai * minimum nécessaire au régulateur optimum pour mener à bonne fin la mancuvre de réglage provoquée par une variation brusque et faible de la puissance consommée.

Le remède employé jusqu'ici consiste à régulariser la pression de l'eau au vannage; il est théoriquement parfait, puisque appliqué intégralement, il supprime la cause des oscillations. Ii convient mal en pratique pour diverses raisons que nous allons rappeler:

Tout d'abord, les appareils mécaniques désignés sous le nom de déchargeurs doivent être écartés: ils sont destinés à assurer la sécurité de la conduite contre les surpressions produites par d'amples manœuvres du vannage, mais ne peuvent intervenir à l'occasion des petits mouvements du régulateur qui suffisent à constituer un pompage gênant.

La solution classique consiste à employer une cheminée ou chambre d'équilibre, capacité ou. verte à l'air libre et placée en principe, au droit du vannage. Elle est destinée à absorber ou compenser les écarts de débit entre conduite et turbine sans occasionner de variation notable de la pression, du moins pendant la durée d'une oscillation du régulateur. Ses déficiences se manifestent dans la réalisation pratique. La cheminée ne peut en fait, être placée très près du vannage; il reste entre elle et lui une «conduite forcée » de longueur au moins égale à la chute, quelle qu'en soit la hauteur, et spécialement en basse chute, souvent notablement supérieure.

On a parfois tenté d'utiliser une capacité fermée, un « réservoir d'air »: celui-ci aurait pu être placé très près du vannage. Malheureusement, les dimensions qu'il devrait avoir sont prohibitives. Une capacité ainsi placée à l'extrémité d'une conduite, réservoir d'air ou cheminée d'équilibre, constitue en effet un oscillateur à longue période dont on ne peut augmenter à son gré l'amortissement; de plus, le jeu du régulateur, maintenant constante la puissance empruntée à la conduite par la turbine, est à l'origine d'un amortissement négatif qui, si la capacité $n^{\prime}$ est pas suffisante, entretient ou même amplifie les oscillations.

L'ingénieur allemand THOMA a le premier dépisté ces phénomènes et indiqué le diamètre minimum à donner à une cheminée d'équilibre en supposant un amortissement dû uniquement à la perte de charge dans le canal d'amenée. Sa formule a été perfectionnée pour convenir à d'autres conditions d'amortissement; on peut l'étendre de manière très simple au cas du réser. voir d'air.

\section{2" Solution proposée}

Analysons le principe de solution qui aboutit aux réalisations dont nous venons de parler. Lo régulation des turbines hydrauliques n'y est pas envisagée directement comme telle, c'est-à-dire en tenant compte du phénomène du coup de bélier. Elle est étudiée par comparaison avec celle d'un groupe où l'inertie du fluide moteur est négligeable, celle d'un groupe à vapeur par exemple. Celle-ci étant plus facile, on tâche d'amenuiser et si possible, de faire disparaitre les différences qui séparent les deux cas ; aussi, cherche-t-on à maintenir constante la pression au vannage.

Notre solution envisage directement le phénomène complexe : réglage de la vitesse lorsque le fluide moteur présente une inertie importante. Le régulateur doit alors être «défendu » contre les effets des variations transitoires de pression qu'il engendre; il comporte donc, outre le tachymètre et l'accéléromètre, un DETECTEUR MANOMETRIQUE DIFFERENTIEL: celui-ci intervient de telle manière $\mathrm{qu}^{\prime} \mathrm{il}$ imprimerait au régulateur, en l'absence des deux autres détecteurs, un mouvement apte à faire disparaître l'écart enregistré par lui entre la pression instantanée et la pression moyenne au vannage; en d'autres termes, plus la surpression (Dépression) dans la conduite est élevée, plus le détecteur manomé. trique différentiel ralentit le mouvement de fermeture (ouverture) du vannage. 
Nous adoptons donc la disposition représentée sur la figure 36. Un tube plissé $L$ est placé l'intérieur d'un réservoir $R$, de telle sorte qu'il supporte extérieurement la pression régnant dे l'intérieur du réservoir. La partie intérieure de $L$ communique par une large tuyauterie $t$ avec un point $V$ convenablement choisi dans la conduite forcée et reste à tout instant soumise a la pression existant en ce point. L'intervalle compris entre $L$. et $R$ contient une certaine masse d'air dont on verra le rôle dans un instant. Sa partie basse communique avec la tuyauterie t par une tubulure de faible diamètre, dont on peut d'ailleurs régler la section utile par un obturateur $r$. Dans ces conditions, au contraire de la partie intérieure du tube plissé, la partie exté. rieure ne se met à la pression régnant au point $V$ que lentement, et en particulier, reste insensible aux variations de cette pression pendant la période d'oscillation du régulateur.



Fig. 36. - Schéma de principe du triple détecteur pour le réglage de vitesse des turbines hydrauliques.

Le fond du tube $L$ est donc soumis sur ses deux faces à des pressions dont la différence est l'écart instantané de pression au point $V$. Cette différence est équilibrée par la force élastique du tube, complétée par celle de ressorts antagonistes non représentés. Le déplacement du fond de $L$ et par suite, ceux de $M^{\prime 2}$ et $M_{2}$ qui lu: sont liés sont donc proportionnels à l'écart de pression au point $V$; ils sont du même coup, proportionnels à l'écart instantané de pression en un point quelconque (abstraction faite des. phénomènes de turbulence) et finalement, constituent des mesures de $\zeta$.
Des dispositions particulières sont prévues pour que la masse $d^{\prime}$ air contenue dans $R$ reste toujours suffisante, et inversement, un orifice capillaire pratiqué au fond de $L$ empêche l'air de s'accumuler à l'intérieur du tube plissé. Le point $V$ est évidemment choisi pour réduire au minimum les anomalies dues à la turbulence.

Le déplacement du point $M_{2}$ s'introduit dans le mécanisme de régulation exactement comme ceux des points $M$ et $M_{1}$, tel qu'il a été exposé au chapitre 1. Tandis que, dans le schéma à double détection de la figure 1, le mouvement du tiroir $T$ était une moyenne pondérée des mouvements de $M_{0}$ et $M_{1}$, ici (figure 36), par le jeu d'un levier flottant supplémentaire, il correspond à une moyenne pondérée des mouvements de $M_{1} M_{1}$ et $M_{2}$.

\section{Equations de la régulation}

Rien n'est changé aux équations données au chapitre.IV, à l'exception de l'équation (11) qui devient :

$$
\frac{\mathrm{d} \varepsilon}{\mathrm{dt}}=-\mathrm{K}_{0} \xi-\mathrm{K}_{1} \frac{\mathrm{d} \xi}{\mathrm{dt}}+\mathrm{K}_{2} \zeta \quad(11 \mathrm{bis})
$$

en désignant par $K_{2}$ le coefficient de réponse manométrique; la résolvante (17) devient alors:

$$
\frac{r^{2}+(A+C) r+B}{\frac{r^{2}}{2}+\left(\frac{C}{2}+D-A\right) r+C D-B}=-2 p \text { th } r(38)
$$

Nous n'étudierons ici, comme aux chapitres précédents, la double influence des termes $C$ et $D$, qu'avec I'hypothèse du mouvement en masse; nous laisserons donc encore de côté l'influence de l'autoréglage lorsque nous étudierons celle de l'élasticité.

La résolvante s'écrira dans le premier cas:

$$
r^{\prime} 3+2\left(1-A^{\prime}+\frac{C^{\prime}}{2}+D^{\prime}\right) r^{\prime 2}+
$$

$2\left(A^{\prime}-B^{\prime}+C^{\prime}+C^{\prime} D^{\prime}\right) r^{\prime}+2 B^{\prime}=0$

en posant par analogie avec ce que nous avons fait précédemment :

$$
D^{\prime}=2 \rho D=K . \theta \text {. }
$$

Dans le deuxième cas, elle sera :

$$
\frac{r^{2}+A r+B}{\frac{r^{2}}{2}+(D-A) r-B}=-2 \rho \text { th } r
$$




\section{4" Condition de stabilité dans l'hypothèse du mouvement en masse}

L'étude qualitative de la stabilité ne présente aucune difficulté nouvelle du fait de l'existence du paramètre $D^{\prime}$. Les conditions d'HURWITZ appliquées à l'équation (39) s'écrivent:

$$
\begin{aligned}
1-A^{\prime}+ & \frac{C^{\prime}}{2}+D^{\prime} \geqslant 0 \quad B^{\prime} \geqslant 0 \\
& 2\left(A^{\prime}-B^{\prime}+C^{\prime}+C^{\prime} D^{\prime}\right) \\
& \left(1-A^{\prime}+\frac{C^{\prime}}{2}+D^{\prime}\right) \supseteq B^{\prime}
\end{aligned}
$$

et la condition (41) qui, seule est à retenir en plus de $B^{\prime} \geq 0$, se traduit :

$$
B^{\prime} \leqslant \frac{\left(A^{\prime}+C^{\prime}+C^{\prime} D^{\prime}\right)\left(2-2 A^{\prime}+C^{\prime}+2 D^{\prime}\right)}{3-2 A^{\prime}+C^{\prime}+2 D^{\prime}}
$$

Lorsque $C^{\prime}$ et $D^{\prime}$ sont domnés, la limite de stabilité peut encore être représentée comme nous l'avons fait jusqu'ici, sur la figure 21 par exemple, par un diagramme en $A^{\prime} B^{\prime}$. La courbe limite reste un arc d'hyperbole. Elle rencontre l'axe des ordonnées aux points:

$$
A^{\prime}=-C^{\prime}\left(1+D^{\prime}\right) \text { et } A^{\prime}=1+D^{\prime}+\frac{C^{\prime}}{2}
$$

Le maximum de $B^{\prime}$ est obtenu lorsque :

$$
A^{\prime}=\frac{3+C^{\prime}+2 D^{\prime}-\sqrt{\left(1+C^{\prime}\right)\left(3+2 D^{\prime}\right)}}{2}
$$

II a pour valeur:

$$
B^{\prime}=\frac{\left(\sqrt{\left(1+C^{\prime}\right)\left(3+2 D^{\prime}\right.}-1\right)^{2}}{2}
$$

De ces seuls résultats, ressortent les conclusions suivantes:

1) l'effet du détecteur manométrique s'ajoute à celui de l'autoréglage pour étendre la zone de stabilité dans toutes les directions, sauf cependant en ce qui concerne la condition $B^{\prime}$ positif qui reste inchangée. En particulier, I'absence d'accéléromètre continue à être tolérable et le maximum de $B^{\prime}$ compatible avec cette absence est relevé.

2) En principe, rien ne s'oppose à l'extension à I'infini de la zone de stabilité, puisque le constructeur peut disposer entièrement du choix de $D^{\prime}$; en pratique, il ne pourra tout de même pas l'augmenter indéfiniment.

Pour visualiser ces résultats, nous avons délimité sur la figure 37 la zone de stabilité dans les cas suivants:

Courbe 1: $C^{\prime}=0, \quad D^{\prime}=0$ (rappel de la figure 20).
Courbe II: $C^{\prime}=0,3, D^{\prime}=0 \quad$ (rappel de la figure 27).

Courbe 111: $C^{\prime}=0, \quad D^{\prime}=15$ Cobtenu par exemple avec $K_{2}=10$ lorsque $\theta=1,5)$.

Courbe IV: $C^{\prime}=0,3, D^{\prime}=15$.



Fig. 37. - Condition de stabilité

I. sans autoréglage ni détection manométrique.

11. avec cutoréglage sans détection manométrique.

III. sans autoréglage, mais avec détection manométrique.

$I V$. avec autoréglage et détection manométrique.

On voit que la zone est considérablement étendue par le dispositif manométrique. Celui-ci permet donc d'augmenter beaucoup les rapidités de réponse tachymétrique $K_{\text {"1, }}$ et accélérométrique $\mathrm{K}$. Toutefois, comme son action propre s'oppose partiellement à celle de ces deux détecteurs, nous ne devons pas nous attendre à ce que la rapidité de réglage soit augmentée dans la même proportion que $K_{\text {" }}$ et $K_{1}$. Ici plus encore que dans les chapitres précédents, les comparaisons ne peuvent avoir un caractère objectif que si elles portent sur des mesures de stabilité. Nous allons donc, comme nous l'avon: fait au chapitre $V$, déterminer l'optimum théorique et l'optimum pratique, puis nous repren. drons l'application numérique, afin de montrer plus concrètement le gain de stabilité réalisé. 


\section{$5^{\circ}$ Optimum théorique dans I'hypothèse du mourement en masse}

L'optimum théorique est caractérisé par le fait que l'équation (39) admet une racine triple - $y^{\prime}$. On a donc:

$$
\begin{aligned}
y^{\prime 3} & =2 B^{\prime} \\
3 \quad y^{\prime 2} & =2\left(A^{\prime}-B^{\prime}+C^{\prime}+C^{\prime} D^{\prime}\right) \\
3 \quad y^{\prime} & =2-2 A^{\prime}+C^{\prime}+2 D^{\prime}
\end{aligned}
$$

Si nous traitions ce système comme celui qui lui correspond au chapitre $V$, il viendrait :

$$
\begin{aligned}
& A^{\prime}=\frac{\gamma^{\prime 3}+3 \gamma^{\prime 2}-6\left(1+D^{\prime}\right) \gamma^{\prime}+4\left(1+D^{\prime}\right)=}{6+4 D^{\prime}} \\
& B^{\prime}=\frac{\gamma^{\prime 3}}{2} \\
& C^{\prime}=\frac{\gamma^{\prime 3}+3 \gamma^{\prime 2}+3 \gamma^{\prime}-2\left(1+D^{\prime}\right)}{3+2 D^{\prime}}
\end{aligned}
$$

Nous tracerions alors une famille de courbes $D^{\prime}=$ Cte graduées en valeurs de $C^{\prime}$ et $D^{\prime}$. La première d'entre elles, $D^{\prime}=0$ serait la courbe 1 de la figure 23. Nous avons préféré donner sur la figure 38 (partie droite) les deux courbes $C^{\prime}=0$ et $C^{\prime}=0,3$ graduées en valeurs de $D^{\prime}$, et reporter en abscisses avec les valeurs de $B^{\prime}$ celles de $\gamma^{\prime}$. Nous avons pour cela écrit le système (42) sous la forme:

$$
\begin{aligned}
& D^{\prime}=\frac{\gamma^{\prime 3}+3 \gamma^{\prime 2}+3 \gamma^{\prime}-2-3 C^{\prime}}{2\left(1+C^{\prime}\right)} \\
& B^{\prime}=\frac{\gamma^{\prime 3}}{2} \\
& A^{\prime}=\frac{y^{\prime 3}+3 \gamma^{\prime 2}-3 C^{\prime} \gamma^{\prime}+C^{\prime \prime}}{2\left(1+C^{\prime}\right)}
\end{aligned}
$$

Nous pouvons maintenant mieux apprécier l'intérêt du dispositif. A la courbe IV de la fig. 37 $\left(C^{\prime}=0,3 ; D^{\prime}=15\right)$ correspond l'optimum théorique $A^{\prime}=12,4 ; B^{\prime}=7,8$ et un amortissement tel que $y^{\prime}=2,5$. A la courbe 11 $\left(C^{\prime}=0,3 ; D^{\prime}=0\right)$ correspondraient seulement, d'après la figure 23 à plus grande échelle, $\mathrm{A}^{\prime}=0,288 ; \mathrm{B}^{\prime}=0,095$ et un amortissement tel que $y^{\prime}=0,575$, donc égal aux $23 / 100 \mathrm{de}$ celui que permet le dispositif envisagé. Le délai de réglage serait donc réduit dans ces conditions à moins du $1 / 4$ de sa valeur actuelle.

\section{6" Optimum pratique dans l'hypothèse du mourement en masse}

Rappelons que nous avons adopté pour opt:mum pratique une combinaison telle que la



Fig. 38. - Amélioration de l'optimum par l'emploi du détecteur manométrique (hypothèse du mouvement en masse).

composante apériodique des mouvements de réglage ait même amortissement que la composonte périodique et qu'en outre, le rapport $n$ de la pulsation de celle-ci à son amortissement soit égal à 2. Nous avons motivé ce choix, spécialement au chapitre $V$, et montré que l'élément subjectif qu'il comporte nécessairement est de minime conséquence. Les trois racines de l'équa. tion (39) doivent être d'après cette définition :

$$
-y^{\prime},-y^{\prime}+i \varphi^{\prime}-y^{\prime}-i \varphi^{\prime}
$$

Mais pour qu'il en soit ainsi, il faut que soient satisfaites les conditions (30) et (31) que nous avons établies au $\$ 6$ du chapitre $V$. Rappelonsles. L'équation étudiée :

$$
r^{\prime} 3+2\left(1-A^{\prime}+\frac{C^{\prime}}{2}+D^{\prime}\right) r^{\prime 2}+
$$

$2\left(A^{\prime}-B^{\prime}+C^{\prime}+C^{\prime} D^{\prime}\right) r^{\prime}+2 B^{\prime}=0$ (39) étant écrite sous la forme abrégée : $f\left(-r^{\prime}\right)=0$, et les symboles $f^{\prime}$ et $f^{\prime \prime}$ désignant respective. ment les dérivées première et seconde par rapport à $r^{\prime}$ de la fonction $f$, il faut et il suffit que I'on ait : 


$$
\begin{aligned}
& f_{\left(-y^{\prime}\right)}=f^{\prime \prime}\left(-y^{\prime}\right)=0 \\
& n^{2} y^{\prime 2}=\varphi^{\prime \prime}=f^{\prime}\left(-\gamma^{\prime}\right)
\end{aligned}
$$

Dans le cas présent, ces conditions s'écrivent :

$$
\begin{gathered}
\gamma^{\prime}-2\left(1-A^{\prime}+\frac{C^{\prime}}{2}+D^{\prime}\right) y^{\prime 2}+ \\
2\left(A^{\prime}-B^{\prime}+C^{\prime}+C^{\prime} D^{\prime}\right) \gamma^{\prime}-2 B^{\prime}=0 \\
3 y^{\prime}=2-2 A^{\prime}+C^{\prime}+2 D^{\prime} \\
n^{2} y^{\prime}=-3 y^{\prime 2}-2\left(2-2 A^{\prime}+C^{\prime}+\right. \\
2 D^{\prime} y^{\prime}+2\left(A^{\prime}-B^{\prime}+C^{\prime}+C^{\prime} D^{\prime}\right)
\end{gathered}
$$

ou encore :

$$
\begin{aligned}
2-2 A^{\prime}+C^{\prime}+2 D^{\prime}=3 & y^{\prime} \\
2\left(A^{\prime}-B^{\prime}+C^{\prime}+C^{\prime} D^{\prime}\right) & =\left(n^{2}+3\right) y^{\prime 2} \\
2 B^{\prime} & =\left(n^{2}+1\right) y^{\prime}
\end{aligned}
$$

que nous traduirons comme au paragraphe précédent :

$$
\begin{aligned}
& A^{\prime}=\frac{\left(n^{2}+1\right) y^{\prime}+\left(n^{2}+3\right) \gamma^{\prime 2}-3 C^{\prime} \gamma^{\prime}+C^{\prime \prime}}{2\left(1+C^{\prime}\right)} \\
& B^{\prime}=\frac{n^{2}+1}{2} y^{\prime 3} \\
& D^{\prime}=\frac{\left(n^{2}+1\right) \gamma^{\prime 3}+\left(n^{2}+3\right) \gamma^{\prime 2}+3 y^{\prime}-3 C^{\prime}-2}{2\left(1+C^{\prime}\right)}
\end{aligned}
$$

La représentation en est donnée dans la partie gauche de la figure 38, où nous avons tracé les deux courbes $C^{\prime}=O$ et $C^{\prime}=0,3$, cotées en valeurs de $D^{\prime}$. Les échelles de $A^{\prime}$ et $y^{\prime}$ sont les mêmes que pour l'optimum théorique, mais les valeurs lues sur l'échelle de $B^{\prime}$ doivent être multipliées par 5 .

Si nous reprenons à titre d'exemple $C^{\prime}=0,3$ et $D^{\prime}=15$ l'optimum pratique correspond à : $A^{\prime}=13,6 ; B^{\prime}=9,75 ; y^{\prime}=1,58$, tandis que la figure 23 indique pour la même valeur de $C^{\prime}$. mais avec $D^{\prime}=0 ; A^{\prime}=0,516, B^{\prime}=0,19$ et $\gamma^{\prime}=0,423$ soit les $27 / 100$ environ du précédent. Le délai de réglage doit donc du fait du détecteur manométrique, être réduit environ au $1 / 4$ de sa valeur actuelle.

Signalons cependant au passage, à quel point il serait erroné d'apprécier l'accélération du réglage par les accroissements de $K_{11}$ et $K_{1}$. Dans l'exemple envisagé, ils sont multipliés res. pectivement par 51 et 26 , tandis que l'amortis. sement est seulement multiplié par 3,7 .

\section{7" Equde des caractéristiques de ce régloge sur un exemple numérique}

L'intégration complète du système différentiel permet ici, comme au chapitre $V \$ \$ 7$ et $\delta$. d'étudier les changements que subissent les di.. verses variables au cours d'une petite manœuvre de réglage caractérisée par une valeur initiale $\varepsilon_{i}$ de l'écart relatif d'ouverture. Les écarts des diverses variables sont évidemment proportionnels à $\varepsilon_{0}$ : l'écart de vitesse $\triangle \omega$ ou sa valeur relative $\xi=\frac{\Delta \omega}{\omega_{0}}$ par le rapport $==\frac{\xi}{\varepsilon_{0}}$; de même, nous avons caractérisé l'écart de pression par $Z=\frac{\zeta}{\varepsilon_{1}}$ et celui d'ouverture par: $E=\frac{\varepsilon}{\varepsilon_{0}}$. Nous avons appelé labilité le maximum de $=$.

Afin de faciliter les comparaisons sans multiplier les calculs, nous reprendrons les mêmes inerties de l'eau : $\theta=3$ et du rotor: $\tau=7,5$, que pour l'exemple a du chapitre $V \S 8$, et le même coefficient d'autoréglage $\alpha=1$. La variation de $=$ lorsque $K_{\text {.. }}$ sera nul sera donc celle qu'indique la courbe a de la figure 25 . Nous l'avons reprise à la figure 39 (Courbe a) à côté de la courbe b représentant la variation de $=$ avec les mêmes données, sauf $\mathrm{K}_{2}=10$ au lieu de $K_{2}=0$. Nous avons en outre tracé la courbe $c$ qui ne diffère de la courbe $b$ que par l'inertie du rotor: $\tau=10$ au lieu de $\tau=7,5$.

On voit que l'adoption du dispositif manométrique sans modification de l'inertie du rotor réduit le délai de réglage approximativement au $1 / 5$ de sa valeur, mais augmente de $25 \%$ la labilité. Une augmentation de $33 \%$ de l'inertie du rotor ramène la labilité à sa valeur première sans détriment de la rapidité du réglage. En somme, tandis que dans la disposition actuelle, on peut seulement réduire la labilité par le jeu de l'inertie $\tau$, dans celle que nous proposons on peut à la fois réduire le délai et la labilité par le double jeu de $K_{\text {. }}$ et de $\tau$. Toutefois, puisque nous avons remarqué (au chapitre $V \S 8$ ). que le choix de $\tau$ peut être dicté par d'autres considérations, nous devons chercher à préciser le gain apporté à la régulation indépendamment de lui par la seule intervention de $\mathrm{K}_{2}$.

Comme on le verra en comparant les figures 40 et 41 , les courbes $a$ et $b$ de la figure 39 sont pratiquement semblables au sens indiqué au chapitre II $\$ 4$. Nous pouvons par suite utiliser les produits des labilités par les racines carrées des délais pour comparer les écarts aléatoires de vitesse des groupes ou ceux des moteurs synchrones qu'ils alimentent, donc aussi les degrés d'irrégularité des machines qui leur sont 


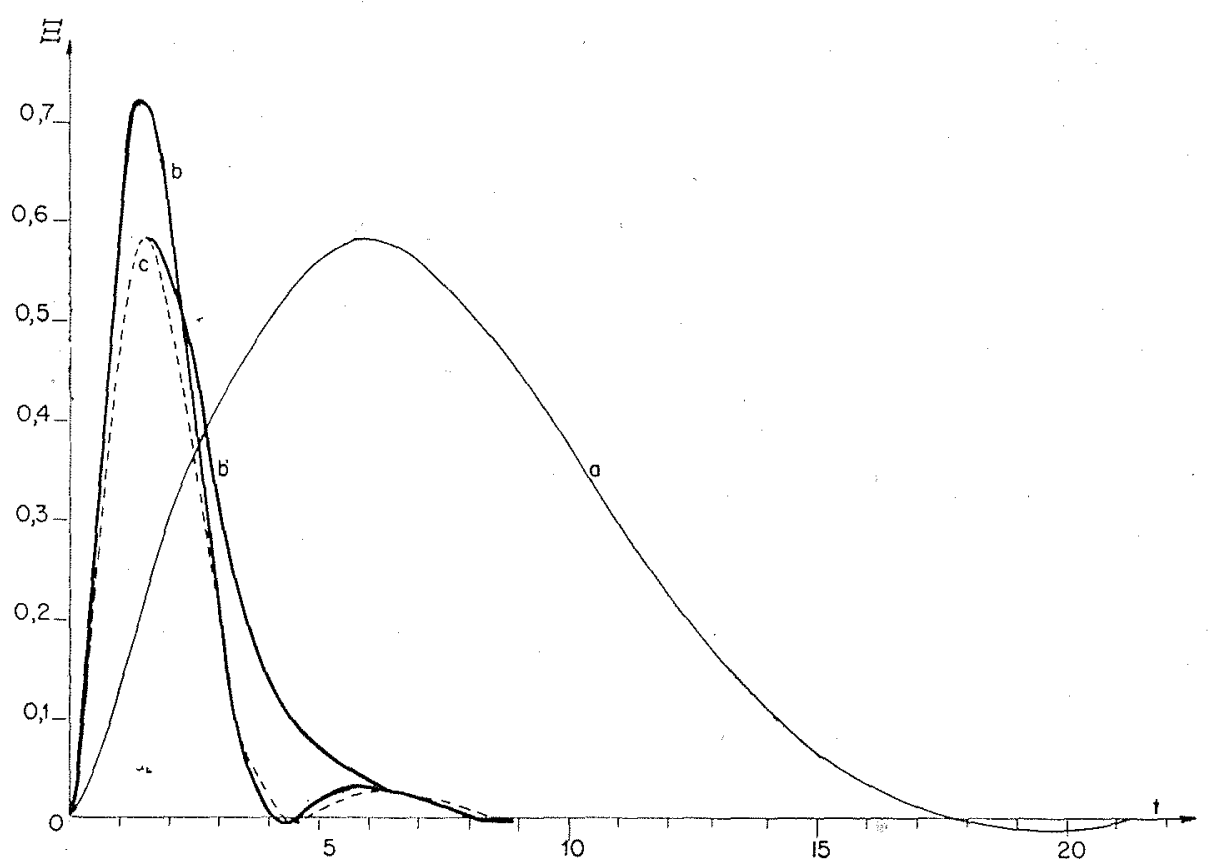

Fig. 39. - Pointe de vitesse au cours d'une petite manœuvre de réglage sur un groupe alimenté par une canalisation à grande inertie : $\theta=3$.

$$
\begin{aligned}
& \text { a- Disposition actuelle } K_{2}=0, T=7,5 \\
& \left(\lambda=0,148 \quad \omega=0,296 \quad K_{0}=0,184\right. \\
& \left.K_{1}=1,33\right) \\
& b-\text { avec détecteur manométrique } K_{2}=10 \quad \tau=7,5 \\
& \left(\lambda=0,71 \quad \omega=1,43 \quad K_{0}=20,1 \quad K_{1}=70\right) \\
& c-\text { avec détecteur manométrique et rotor alourdi } \\
& K_{2}=10 \quad \mathrm{~T}=10 \quad(\lambda=0,69 \\
& \left.\omega=1,38 \quad K_{0}=24,7 \quad K_{1}=93,5\right)
\end{aligned}
$$

liées rigidement. Dans notre exemple, l'irrégu-

$$
\text { larité est divisée par } \frac{\sqrt{5}}{1,25}=1,8 \text {. }
$$

Par ailleurs, une machine qui exige une très grande régularité peut être pourvue d'un volant important et accouplée élastiquement au moteur synchrone qui l'entraine. Dans la mesure où ce dispositif est efficace, il la soustrait aux écarts instantanés de fréquence du réseau, mais la laisse soumise aux écarts cumulés ou écarts horaires. Or, nous avons vu que ceux-ci sont liés au produit de la labilité par le délai et non plus par sa racine carrée. Le gain de régularité apporté à de telles machines en munissant les générateurs du réseau de la triple régulation proposée est donc supérieur à celui dont bénéficient les machines à liaison rapide. A la limite, il passerait de 1,8 à 4 dans notre exemple.

L'existence de ce gain supplémentaire peut s'expliquer de manière intuitive, si l'on remarque que la triple régulation ne réduit pas seulement l'importance globale des irrégularités de vitesse du groupe, mais aussi leur durée individuelle, circonstance avantageuse, car elle diminue leur influence sur les récepteurs. CONCLUONS: Dans notre exemple, la qualité du réglage de vitesse est multipliée par 1,8 pour tous les utilisateurs, et pour les plus exigeants, par un nombre plus élevé tendant vers 4 dans les conditions les plus favorables.

Par ailleurs, la remarque faite à la fin du paragraphe 6 peut être renouvelée ici : le passage de la courbe a à la courbe b correspond aux mul. tiplications de $K_{0}$ par 109 et de $K_{1}$ par 52. tandis que $\lambda$ et $(\omega)$ ne sont multipliés que par 4,8 . On notera aussi que pour une valeur de $K$ donnée $\left(K_{2}=10\right.$ dans le présent exemple comme dans celui du paragraphe 6), l'amélioration apportée par le détecteur manométrique est d'autant plus grande, même en valeur relative, que l'inertie de l'eau est plus élevée. Nous avons pu, par exemple, diviser le délai par 4,8 dans le 
dernier cas où $\theta$ atteignait 3 tandis que nous ne l'avions divisé que par 3,7 dans le cas précédent où $\theta$ était limité à 1,5.

Si suggestive que soit la figure 39, elle ne donne qu'une idée imparfaite du fonctionnement du dispositif proposé; elle en indique le résultat tangible, mais n'en montre pas le mécanisme. Pour mettre celui-ci à découvert, nous avons représenté sur les figures 40 et 41 , outre les $d E$ variations de $=$, celles de : $Z, E$, et $\frac{d E}{d t}$ pour les deux cas étudiés comme types.
a) $K_{z}=0 ; \tau=7,5 ; \theta=3 ; \alpha=1$
(Fig. 40;

a) $\mathrm{K}_{2}=10 ; \tau=7,5 ; \theta=3 ; \alpha=1$

(Fig. 41)



Fig 40. - Evolution au cours d'une manceuvre de réglage, dans les conditions actuelles, de diverses variables : vitesse, ouverture, pression.

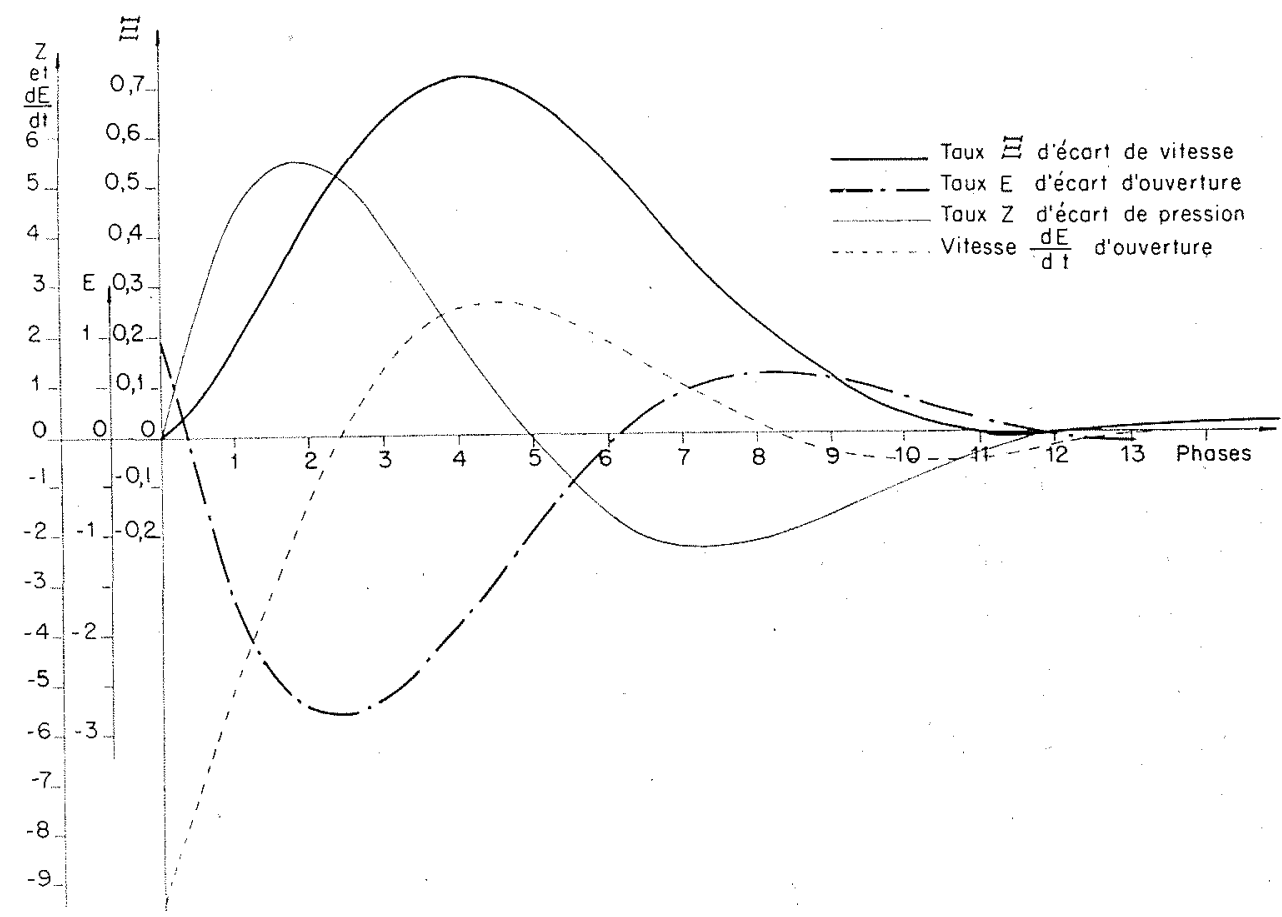

Fig. '41. - Evolution au cours d'une manceuvre de réglage, avec détecteur manométrique, de diverses variables : vitesso, ouverture, pression. 
Les échelles de temps ont été prises différentes, de manière que les délais de réglage, bien que très différents, soient représentés par la même longueur. De même, les échelles des ordonnées ont été choisies diversement, pour que les deux figures restent facilement lisibles.

La comparaison des phases relatives des diverses évolutions représentées sur ces deux figures montre que l'utilisation du détecteur manométrique:

1) retarde légèrement le maximum de l'écart de vitesse et avance très légèrement celui do l'écart de pression, effets qui sont à peu près sans importance.

2) mais avance considérablement le mouve.. ment du vannage. Tandis que, dans la disposition classique, la vitesse de celui-ci est relativement faible au début et croit ensuite pendant un certain temps, l'écart de vitesse ajoutant progressivement son influence propre à celle de l'accélération, dans la disposition envisagée, le mouvement du vannage atteint au contraire son maximum de rapidité dès le début de la manceuvre et se ralentit ensuite beaucoup lorsque l'écart $\zeta$ de pression de l'eau s'élève. Cette circonstance est très heureuse. II est en effet rationnel d'utiliser une grande vitesse de vannage dès les premiers instants de la manœuvre, alors que l'écart de pression est encore faible.

La comparaison des valeurs numériques esi également intéressante: ses résultats s'expiiquent par la remarque précédente. Du fait que le mouvement du vannage a lieu très tôt, il peut être beaucoup plus ample et surtout plus rapide. Ainsi, sur la figure 39, E passe de 1 à - 0,21, puis revient vers 0 ; sur la figure 40 , il passe de 1 à $-2,8$, ce qui représente un déplacement du servo-moteur trois fois plus grand. Ce déplacement est d'ailleurs obtenu en 10 fois moins de temps, donc trente fois plus vite en moyenne, et même soixante fois plus vite au début. La variation de pression $Z$, il est vrai, atteint sur la figure $40: 5,6$ contre 0,59 sur la figure 39 , mais c'est là un rapport nettement inférieur à celui des vitesses d'ouverture, précisément grâce à l'utilisation rationnelle des premiers instants.

On ne doit d'ailleurs pas attacher autrement d'importance à cette valeur de $Z$, car il ne s'agit, rappelons-le, que de petits mouvements au cours desquels par hypothèse, $\zeta$ n'atteint pas une valeur dangereuse pour la conduite forcée ou pour la bâche de la turbine. L'étude de la stabilité n'a pas à considérer autre chose; les moyens dont elle envisage la mise en cuvre ne le lui permettent pas en général. Les questions de résistance des matériaux et de sécurité au cours des grands mouvements relèvent d'une étude particulière dans laquelle on peut faire état de moyens spéciaux qui seraient sans intérêt au point de vue de la stabilité : le déchargeur par exemple.

Remarque. - Les deux fonctions : stabilisation des mouvements de réglage et garantie de sécurité, sont véritablement distinctes; les confondre serait sans aucun doute une erreur sérieuse. Rien n'empêche toutefois de confier à un même dispositif les deux fonctions, si du moins, il est apte à les assumer l'une et l'autre; la chambre d'équilibre a été pendant assez longtemps et reste encore dans plusieurs cas, le moyen le plus économique, précisément parce qu'ambivalent. Mais cette heureuse circonstance ne doit pas faire oublier la distinction qu'elle ne voile que par une sorte de hasard. En particulier, lorsque l'aménagement d'une chambre d'équilibre efficace n'est pas possible, l'appareil qui doit la remplacer est suivant les cas, soit un déchargeur si les difficultés concernent seulement la sécurité, soit un détecteur manométrique stabilisant la régulation, si c'est au contraire le pompage qui est à craindre, soit même l'ensemble des deux, si l'on est en présence de difficultés des deux ordres.

\section{8" Condition de stabilité en haute chute}

Lorsque la conduite est trop longue pour que I'hypothèse du mouvement en masse puisse être gardée, ce qui est toujours le cas en haute chute, l'étude qualitative de la stabilité doit être faite à partir de l'équation résolvante complète :

$$
\frac{r^{2}+A r+B}{\frac{r^{2}}{2}+(D-A) r-B}=-2 \rho \text { th } r
$$

Cette équation doit n'avoir aucune racine d̀ partie réelle positive. Nous l'avons déjà examinée au chapitre VI dans le cas particulier où le paramètre $D$ est nul; les résultats essentiels de cet examen ont été résumés sur les figures 28 et 29 .

Nous avons signalé alors comment les deux figures présentent chacune un aspect de l'influence des variations du paramètre o d'ALLIEVI. La figure 28, ou toute autre présentation où seraient utilisés les paramètres $A=\frac{K_{1} L}{\tau a}$ et $B=\frac{K_{1} L=}{\tau a^{2}}$, montre bien I'influence d'une modification de o par variation de la vitesse $V$ de l'eau, à longueur de 
canalisation donnée. Pour rendre compte de l'influence de ta hauteur de chute, en pratique, un diagramme en $A^{\prime}=\frac{K_{i} \theta}{\tau}$ et $B^{\prime}=\frac{K_{0} \theta^{2}}{\tau}$ tel que celui de la figure 29, est plus utile parce que dans une comparaison de ce genre, c'est une première approximation moins grossière de regar. der $\theta$ comme constant, plutôt que L. Une étude serrée de cette question, avons-nous dit, ne peut considérer o comme seul variable avec la chute, mais aussi tenir compte des modifications de $\theta$.

L'introduction du détecteur manométrique complique encore la présentation des résultats, puisqu'elle ajoute un nouveau paramètre indépendant. Nous sommes amenés en principe, d̀ tracer pour chacune des valeurs du nouveau. paramètre, une série de courbes analogue à celle de l'une des figures 28 ou 29 , mais 29 de préférence, en raison des propriétés que nous venons de rappeler. Pour simplifier, nous nous contenterons d'une de ces séries; les mêmes raisons qui nous ont fait utiliser comme coor. données $A^{\prime}$ et $B^{\prime}$ au lieu de $A$ et $B$, ou $K$,


pour la nouvelle série $D^{\prime}=K_{2} \theta$ et non $D=\frac{K_{2} L}{a}$ ou $K_{e}$.

C'est pourquoi nous avons dessiné sur la figure 42 dans les mêmes coordonnées $B^{\prime}, A^{\prime}$ que sur la figure 29, mais à une échelle différente, des courbes limites de stabilité, répondant toutes à une même valeur $D^{\prime}=15$; les valeurs de $\rho$ adoptées chacune pour une courbe, sont celles qui ont déjà été prises pour établir la figure 29.

La méthode de détermination des courbes est la même ici qu'au chapitre VI; elle dérive de celle de NYQUIST; nous n'en reprendrons pas l'exposé, mais nous en indiquerons brièvement les étapes. Remplaçant dans l'équation (40) l'inconnue $r$ par $\varphi \mathrm{i}$, on écrit :

$$
\frac{A \varphi+\left(\varphi^{2}-B\right) i}{\frac{\varphi^{2}}{2}+B-(D-A) \varphi i}
$$

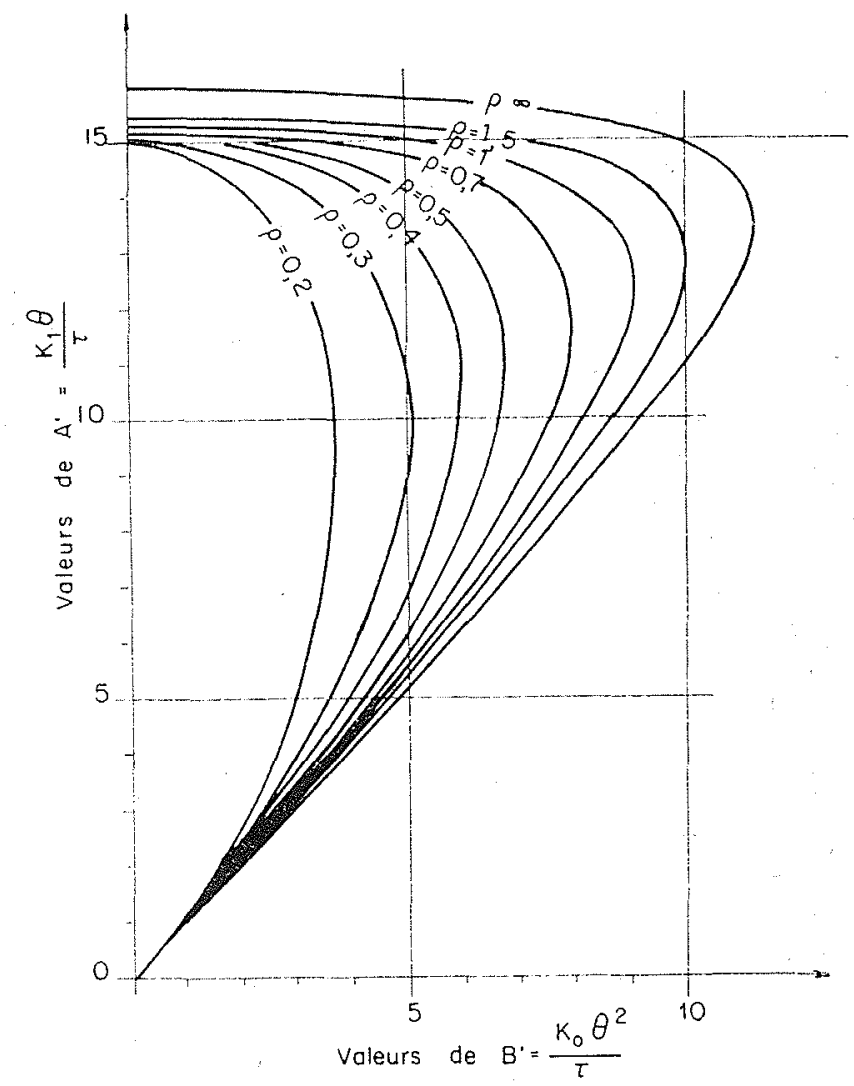

Fig. 42, - Limite de stabilité tenant compte de l'élasticité et des effets du détecteur manométrique. On a pris $D^{\prime}=15$ pour toutes les courbes. 
Le régime éolien est caractérisé par les deux équations :

$$
\frac{A \varphi}{\varphi^{2}+2 B}=\frac{B-\varphi^{2}}{2(D-A) \varphi}=\rho \operatorname{tg} \varphi
$$

et ce système se résout en $A^{\prime}$ et $B^{\prime}$ sous les formes :

$$
\begin{gathered}
A^{\prime}=\frac{4 \rho^{2} \varphi \operatorname{tg} \varphi}{1+4 \rho^{2} \operatorname{tg}^{2} \varphi}\left(D^{\prime} \frac{\operatorname{tg} \varphi}{\varphi}+\frac{3}{2}\right) \\
B^{\prime}=\frac{4 \rho^{2} \varphi \operatorname{tg} \varphi}{1+4 \rho^{2} \operatorname{tg}^{2} \varphi}\left(D^{\prime}+\frac{\varphi}{\operatorname{tg} \varphi}\left(1-2 \rho^{2} \operatorname{tg}^{2} \varphi\right)\right)
\end{gathered}
$$

dont l'exploitation ne présente aucune difficulté.

Le simple rapprochement des figures 25 et 42 montre que le détecteur manométrique joue son rôle, qu'il s'agisse d'installations de haute ou de basse chute. L'augmentation de $\mathrm{B}^{\prime} \mathrm{qu}^{\prime i l}$ permet reste du même ordre dans les différents cas. L'augmentation relative de $A^{\prime}$ est nette.ment plus importante en haute chute puisque le maximum de $A^{\prime}$ reste quasi-indépendant de $o$.

\section{$9^{\circ}$ Exemple d'étude quantitative en haute chute}

La comparaison. par laquelle nous avons conclu l'étude qualitative porte seulement sur les coefficients de réponse $K_{1}$ et $K_{0}$ ou les expressions $A^{\prime}$ et $B^{\prime}$ qui les représentent. Elle est intéressante, mais n'a pourtant pas une valeur absolument décisive.

On peut se demander en particulier, comme nous l'avons fait précédemment, si le détecteur manométrique, en produisant un effet opposé à celui des deux détecteurs cinématiques, n'annule pas les conséquences des augmentations de $K_{0}$ et $K_{1}$ qu'il rend possibles. II les diminue certainement. Nous l'avons déjà observé dans l'hypothèse du mouvement en masse au $\$ 7$ ci-dessus, tout en notant que le résultat restait appréciable, puisque la célérité du réglage devenait environ cing fois plus grande. Mais tandis que dans I'hypothèse précédente, rien ne limitait la pulsation du mouvement de réglage, ici nous avons observé que le mouvement auto-entretenu a toujours une période plus longue que la période propre de la conduite: celle-ci constitue pour elle une limite. Le dispositif manométrique permet bien de la rapprocher de cette limite, non de la lui faire franchir. Faut-il conclure pour autant que le réglage ne peut en aucun cas être obtenu dans un délai moindre? S'il en était ainsi, I'intérêt du dispositif manométrique serait beaucoup moindre dans les installations de haute chute.
En fait, la prépondérance de l'oscillation fondamentale dans les régimes peu amortis disparait lorsque l'amortissement augmente. II subsiste bien un mouvement ayant une période voisine de la période propre de la conduite, mais il cesse d'être le plus important, ainsi qu'on va le voir par l'exemple suivant :

Nous avons cherché par la méthode indiquée au chapitre VI $\S \S 6$ et 7 les pulsations et amortissements des composantes du mouvement libre en utilisant, autant que possible, les mêmes données numériques qu'aux exemples déjà donnés. Nous avons donc adopté : $o=0,3 ; H K_{2}=$ 10. Les valeurs de $\theta$ de 1,5 secondes et plus encore, de 3 secondes, sortiraient ici des limites ordinaires; aussi admettons-nous $\theta=0,9$ et par suite: $L / a=\frac{\theta}{2 \rho}=1,5$, chiffres qui représentent mieux les valeurs couramment réalisées. La valeur de $D^{\prime}=K_{2} \theta$ s'établit alors à 9 et celle de D à 15 .

Nous ne pouvions plus ici pour déterminer l'optimum faire comme précédemment, abstraction de tous les harmoniques de l'oscillation fondamentale; nous devions tenir compte du premier d'entre eux, et, dans une certaine mesure, du second. Il eût d'ailleurs fallu pour préciser cette mesure, recourir à une résolution complète allant jusqu'au tracé de courbes de variation de vitesse, analogues à celles de la figure 39 par exemple. Un contrôle expérimental eût même été préférable et, à vrai dire, seul dirimant, car nos équations conviennent mál à l'étude des fréquences un peu élevées.

Nous pensons pourtant avoir suffisamment approché l'optimum pratique, pour pouvoir formuler quelques remarques. Nous avons choisi : $A=7$ et $B=1,9$ et calculé dans ces conditions pour les premières racines:

$$
\begin{array}{llll}
\varphi_{11}=0,262 & \varphi_{1}=1,86 & \varphi_{3}=4,97 & \varphi_{: 3}=8,09 \\
& & & \varphi_{:}=11,2 \\
\gamma_{0}=0,70 & \gamma_{1}=1,53 & \gamma_{2}=0,67 & \gamma_{:}=0,49 \\
& & \gamma_{i}=0,43
\end{array}
$$

de telle sorte que l'omortissement du premier harmonique se trouve sensiblement égal à celui du mouvement quasi-apériodique $\left(\gamma_{2} \leqslant \gamma_{0}\right.$ et que l'oscillation fondamentale étant beaucoup plus amortie, puisse elle aussi, être considérée comme quasi-apériodique :

$$
\left.l_{1}=\frac{\varphi_{1}}{\gamma_{1}}<2\right)
$$

II est intéressant de rapprocher le délai de 
réglage atteint dans ces conditions du délai minimum exigé en l'absence de détecteur manométrique, et étudié ci-dessus sous le nom d'optimum au chapitre VI $\$ 8$, pour la même valeur 0,3 de $o$. Nous avions alors trouvé $\gamma_{0}=0,333$ et $y_{1}=0,330$ et laissé de côté les amortissements moindres des harmoniques. Les amortissements qui doivent être retenus ici sont largement doubles de ceux-là. Nous pouvons donc estimer le délai de réglage réduit de plus de moitié par l'emploi d'un détecteur manométrique caractérisé par $K_{2}=10$.

Nous ne pouvons donner ce résultat que comme une indication numérique liée à la valeur de $\mathrm{K}_{2}$ choisi avec un certain arbitraire. Rien $n$ 'indique qu'une valeur plus élevée de ce coefficient de réponse ne saurait être admise; le coefficient de réduction du délai en serait accru comme il l'est dans le cas du mouvement en masse (fig. 38).

Mais, et c'est la raison que nous avons donnée du présent examen, il est plus instructif de rechercher si le dispositif a dans les hautes chutes une efficacité égale ou comparable à celle qu'il atteint dans les basses. Nous avons obtenu ici un rapport de réduction du délai de 2 , tandis que nous avions trouvé avec la même valeur de $K$, et dans I'hypothèse du mouvement en masse, un rapport de 3,7 lorsque $\theta$ était de 1,5 (cidessus, $\$ 6$ ) et un rapport de 4,8 lorsque $\theta$ s'élevait à trois secondes $(\S 7)$ : La différence est importante. Elle tient en partie au système de mesure adopté pour caractériser le dispositif manométrique. Ce système a sa logique puisque $K$. est bien un paramètre propre au dispositif. Pourtant, tout comme dans la comparaison instituée au chapitre VI $\$ 2$ entre les conditions de stabilité de haute et de basse chute, il était préférable d'envisager comme première approximation $\theta$ constant plutôt que $\mathrm{L} / \mathrm{a}$ constant; ici, il vaut mieux caractériser le dispositif nouveau par le produit $D^{\prime}=K^{\prime} \theta$ plutôt que par $K_{2}$. C'est en effet à valeur constante de $D^{\prime}$ et non de $K$, que l'on peut parler même dans la simple hypothèse du mouvement en masse, d'un coefficient de réduction du délai : la figure 3.8 le montre immédiatement. Les coefficients de 4,7 et de 3,8 notés tous deux dans cette hypothèse du mouvement en masse, diffèrent parce qu'ils répondent à des valeurs de $D^{\prime}$ différentes, 15 et 30 , malgré l'identité de $K_{2}$ dans les deux cas : $K_{2}=10$. Le rapport de 2 en haute chute est obtenu avec $K_{2}=10$, mais comme $\theta$ n'est que de 0,9 , la valeur de $D^{\prime}$ est seulement de 9 .

Pour la même valeur de $D^{\prime}$ en basse chute, on n'atteindrait pas 4,8 , ni même 3,7 , mais seulement 3,4. La différence d'efficacité du dispo- sitif en hautes et basses chutes est donc un peu moindre qu'il ne pouvait sembler tout d'abord. Elle est loin d'être prohibitive: elle n'en reste pas moins réelle.

Enfin, pour aider à décrire le fonctionnement de ce système de régulation à trois détecteurs, nous attirons l'attention sur la valeur élevée de l'amortissement du mouvement dont la pseudopériode est voisine de la période propre de la conduite. Le retour vers la turbine de l'onde de pression engendrée au début du processus de réglage ne cause à peu près aucun trouble: Ce fait tient à ce que le mécanisme régulateur réagit aux variations de pression dans la conduite directement, et dans un sens propre à les annuler tandis qu'en l'absence de dispositif manométrique, il réagit seulement par l'intermédiaire des détecteurs cinématiques et dans un sens tel qu'il accentue plutôt l'écart de pression qu'il ne le diminue.

\section{CONCLUSIONS}

L'une des principales difficultés de l'étude des phénomènes de régulation réside dans l'ambiguité que comporte la notion de stabilité. La définition habituelle en starique semble n'avoir égard qu'à l'effet de rappel. La notion correspondante en matière de régulation porterait alors le nom de promptitude et se concrétiserait dans les coefficients de réponse $K_{11}$ et $K_{1}$.

L'amortissement que contient de manière implicite la notion de stabilité est en pratique nécessaire, mais apparaît peu ou point dans la définition classique. Au contraire, dans les problèmes de régulation, il iend à être regardé comme constituant à lui seul la stabilité et semble être opposé à la promptitude.

Enfin, le rôle propre de l'inertie qui est « d'étouffer » les perturbations en diminuant l'amplitude de leurs effets n'est pas d'ordinaire dégagé : on estime qu'accroître l'inertie permet $d^{\prime}$ augmenter presque indéfiniment une qualité, la promptitude, sans détriment de la qualité opposée, la stabilité.

Nous avons examiné de plus près ce que couvre la notion de stabilité, et trouvé qu'elle a concrètement le même contenu, qu'il s'agisse de stabilité classique ou de régulation. Ce contenu peut être analysé du point de vue de la cause de la stabilité; on doit alors distinguer comme 
nous venons de le faire, trois éléments tels que : force de rappel, amortissement, inertie, ou d'autres qui leur sont équivalents. Un rappel trop énergique devant l'amortissement provoque des oscillations qui ne s'éteignent que lentement; in amortissement trop fort par rapport au rappel gêne aussi le retour à l'équilibre: un dosage convenable de l'un par rapport à l'autre est donc nécessaire. L'inertie a au contraire un rôle plus séparé.

Le contenu de la notion de stabilité peut aussi être analysé du point de vue des effets. On y trouve encore trois éléments, mais on peut dans une certaine mesure, les ramener à deux. Le premier des trois correspondrait à la proportion «rappel-amortissement » et pourrait être appelé le mode ou la forme du retour à l'équilibre. II est bien caractérisé par le décrément, rapport de deux écarts séparés par un intervalle de temps égal à une pseudo-période. II a été pris comme critère de stabilité par ceux qui ont regardé la pseudo-période comme fixée «ne varietur» par la nature des choses. Nous n'avons pas cru devoir le retenir comme essentiel parce que, parmi les diverses formes ainsi qualifiées, une est à juste titre reconnue comme supérieure aux autres, et définie assez nettement pour constituer un bon terme de référence : elle correspond à un décrément logarithmique de l'ordre de $\pi$ soit à $n=2$ dans nos notations. Lorsque, comme il arrive à partir d'une certaine hauteur de chute, la pseudo-période est plus rigidement imposée, et que de ce fait, le décrément ne peut être aussi élevé qu'on le voudrait, la mesure de ce dernier reprend de l'intérêt.

Les deux autres éléments de la stabilité envisagée dans ses manifestations étaient plus importants pour l'ensemble de notre étude : I'un que nous avons appelé DELAI DE REGLAGE ou simplement délai est le temps nécessaire pour le retour ò l'équilibre après une petite perturbation; I'autre auquel nous avons donné le nom de LABILITE, mesure l'importance de l'écart subi relativement à la cause qui l'a provoqué. Ensemble, ils caractérisent bien le mécanisme de la régulation et constituent des renseignements précis et objectifs d̀ son sujet. Ils sont en outre en rapport étroit avec les causes. L'inertie n'agit que sur la labilité contrairement à ce que l'on semble admettre couramment en matière de régulation; le délai ne dépend que de l'intensité de l'ensemble «rappel-amortissement », la pro- portion entre rappel et amortissement étant par ailleurs déterminée pour réaliser le meilleur mode de retour à l'équilibre. Ces précisions apportées à la notion de stabilité nous ont permis de réduire l'opposition artificielle promptitude-stabilité qui ne répondait ni à la conception commune de la stabilité ni aux « conditions » mathématiques telles que celles d'HURWITZ.

Ces précisions nous étaient apparues nécessaires à une étude quantitative sérieuse de la stabilité des systèmes de régulation, ou en d'autres termes, à l'étude de leur rapidité de réglage. L'étude purement qualitative de la stabilité devait aussi être achevée; M. ALMERAS ne l'avait abordée que pour le cas des basses chutes; M. GADEN l'avait bien envisagée d'une manière générale, mais avait employé un moyen qui ne lui permettait pas de présenter des résultats nets. Nous avons complété, d'abord ces travaux.

Nous avons pu en général définir un optimum de la régulation ayant valeur objective. Le délai nécessaire au réglage dans ces conditions est le minimum pour chaque cas: il caractérise bien les difficultés de régulation propres au cas. Nous avons alors situé cette sorte de «mur » que présente l'inertie de l'eau à l'accroissement de la rapidité de réglage; nous avons même montré dans quelle mesure en hautes chutes, les conséquences de l'inertie sont accentuées du fait de l'élasticité.

Enfin nous avons présenté un moyen apte à réduire, en principe autant qu'on le veut, l'influence de l'inertie de l'eau sur le délai de réglage. La méthode qui nous avait servi à chiffrer les inconvénients enregistrés nous a permis pareillement de préciser, en fonction de l'intensité du remède employé, le gain qu'il procure. Ce gain se présente comme la réduction de délai de réglage dans un rapport donné. Avec uné intensité du remède qui paraît facilement réalisable en pratique, nous avons calculé une réduction de délai atteignant 50 à $65 \%$ dans les installations de hautes chutes et 70 à $80 \%$ dans celles de basses chutes. Ces résultats sont très appréciables, spécialement si l'on a égard à la simplicité du dispositif mécanique utilisé.

Je suis heureux de pouvoir exprimer ici à M. ESCLANGON, Directeur de l'Institut Polytechnique de Grenoble, toute ma reconnaissance 
pour l'aide bienveillante qu'il m'a apportée dans la préparation de cette thèse. Je remercie vivement $M$. FORTIER qui a bien voulu assumer la direction de mon travail et $M$. SILBER dont les conseils m'ont été précieux. Je dois également un merci tout particulier à $M$. LEMAIRE, Directeur de l'Ecole Centrale Lyonnaise, ainsi qu'aux Directeurs et aux Ingénieurs des Ateliers NEYRET-BEYLIER ET PICCARD-PICTET, spécialement MM. GARIEL, Président Directeur Général, DANEL, ALMERAS et CHENAIS qui ont mis à ma disposition leur abondante documentation et $\mathrm{m}$ 'ont fait largement profiter de leur expérience. Mes remerciements vont aussi aux industriels, constructeurs et exploitants que j'ai pu consulier et dont les enseignements $m^{\prime}$ ont été précieux, en particulier à M. DARRIEUS, membre de I'Institut, Ingénieur-Conseil à la Compagnie Electro-Mécanique, MM. LAURENT et VILAIN, Ingénieurs de la Maison RATEAU, M. GADEN, Directeur des Ateliers des Charmilles, MM. les Ingénieurs de l'Electricité de France THALLER, DUPRE LA TOUR, ROBERT, BONNEFOY à Lyon, GIRON et CABROL au Puy.

Enfin, je remercie le R.P. TALPAIN du concours efficace qu'il m'a apporté, tout particulièrement en ce qui concerne les documents de langue allemande.

\section{PRINCIPAUX OUVRAGES CONSULTES}

1. - P. Allleret, Ingénieur des Ponts \& Chaussées : Cours d'Electrotechnique appliquée (Ecole Nationale des Ponts \& Chaussées 1942-1943).

2. - P. AILLERET: Le réglage de la fréquence et de la puissance (Cours à l'Ecole supérieure d’Electricité 1946).

3. - L. ALlievI (Traduction OGUEY): Le coup de bélier et le réglage automatique des turbines hydrauliques. Revue générale de l'Electricité, t. 33, $n^{0} 26$ (1 $1^{\mathrm{er}}$ juillet 1933), pp. 847-859.

4. - P. ALMERAS, ancien élève de l'Ecole Polytechnique, Ingénieur aux Ateliers NEYRET, BEYLIER \& PICCARD-PICTET : Influence de l'inertie de l'eau sur la stabilité d'un groupe hydroélectrique. "La Houille Blanche $»$, Nlle série, $n^{\circ} 1,2,3,5,6$ (Nov. 1945 à Nov. 1946).

5. - P. ALMERAS : Etude de la stabilité des groupes générateurs hydroélectriques interconnectés en tenant compte de l'inertie de l'eau dans les conduites forcées (Revue générale de l'Electricité, t. 56, no 1 (Janv. 1947), pp. 47-50.

6. - P. BABLON, ancien élève de l'Ecole Polytechnique La régulation des groupes hydroélectriques. Revue d'électricité et de mécanique (Alsthom, Mars-Avril 1936).

7. - L. BARBILLION, Directeur de I'Institut Polytechnique de l'Université de Grenoble, Professeur à la Faculté des Sciences. Les groupes électrogènes ef leurs régulateurs (Albin-Michel, Paris 1928).

8. - L. BERGERON : Méthode graphique générale de calcul des propagations d'ondes planes, Mémoires de la Sociéré des Ingénieurs Civils de France, 90" année, fasc. 4 (Juill.-Août 1937).

9. - A. BLONDEL : Sur les systèmes à oscillations persis tantes et en particulier sur les oscillations entrerenues par auto-amorçage. Journal de Physique, 5 série, t. 9 (Avr. 1919, pp. 117-151).

10. - J. CALAME \& D. GADEN : Théorie des chambres d'équilibre, Editions La Concorde, Lausanne 1926 et Gauthier Villars, Paris 1928.

11. - P. CAYERE : Les régulateurs de vitesse des turbines hydrauliques. Arts \& Métiers, $n^{\circ} 84.85$. (Sept.Oct. 1927), pp. 317-326, 357-370.

12. - J. CHENAIS, Ingénieur E.S.E. et P.T.T., ingénieur aux Ateliers N.B.P.P. ; Cours de régulation des turbines hydrauliques et des réseaux d'interconnexion. Ecole des Ingénieurs Hydrauliciens de Grenoble, 1945.

13. - DEN HARTOG (Traduction SUPPER) : Vibrations et mouvements vibratoires, Dunod, Paris 1936.

14. - J. FALLOU, Docteur ès-Sciences, Ingénieur en chef à l'Union d'Electricité : Leçons d'Electrotechnique générale professées à l'Ecole supérieure d'Electri. cité. Gauthier Villars, Paris 1939.

15. - R. FEISS : Détermination de la stabilité des régulateurs au moyen du diagramme vectoriel. Zeitschrift des Vereines deutscher Ingenieure 126 oct. 1940), p. 819

16. - Docteur de FREUDENREiCH : Stabilité des systèmes de réglage. Document inédit de la Société suisse Brown-Boyeri.

17. - D. GADEN, Directeur des Ateliers des Charmilles, Genève: Contribution à l'étude des régulateurs de vitesse. Considérations sur le problème de la stabilité, plus particulièrement dans le cas d'une 
turbine hydraulique dont le système d'alimentation est soumis au phénomène dú coup de bélier. - La Concorde, Lausanne 1945.

I8. - D. GADEN, A. GERMOND, Th. BOVET et J. DES BAILLETS : Etude de la stabilité d'un réglage automatique de vitesse par des diagrammes vectoriels en vue de I'utilisation des critères do NYQUIST. Informations techniques. Charmilles, n' 2, Genève 1946.

19. - J. GRANIER Professeur à la Faculté des Sciences de Besançon : Les régulateurs. Dunod, Paris 1939.

20. - L. LE BLAN : La régulation automatique de haute précision. Mesures no 98 (Juill. 45) . pp. 429-435.

21. - R. LEGENDRE, Ingénieur en chef du Génie Maritime: Principes de régulation. Bulletin de l'Association technique maritime et aéronautique $n^{\circ} \cdot 44$ (1945), pp. $313: 353$.

22. - P. LEMAIRE, Docteur ès-Sciences, Directeur de l'Ecole Centrale Lyonnaise: Enseignement de la physique du mouvement. Le mouvement périodique $(2$ vol.).
23. - P. LEMAIRE : Compléments à I'outillage moderne de I'Ingénieur, Ire partie, Calcul d'HEAVISIDE.

24. - A. LUTHI: Les oscillations des régulations et les diagrammes vectoriels obliques. Schweizerische Bouzeitung 11, avril 1942, pp. 171-174.

25. - NYQUIST : The regeneration theory The Bell System technical. Journal 1932, pp. 126-147.

26. - Y. ROCARD, Docteur ès-Sciences: Les phénomènes d'auto-oscillation dans les installations hydrauliques. Hermann (Actualités scientifiques et industrielles $n^{\circ}$ 544, (X), Poris 1937

27. - Y. ROCARD : Dynamique générale des vibrations, Masson Paris 1943.

28. - Th. STEIN: Etude systématique des régulateurs. Bulletin Escher-Wyss, t. 13 (1940), pp. 59-64.

2.9. - A. TENOT:" Turbines hydrauliques et régulatcurs automatiques de vitesse. Eyrolles Paris 1935



